\title{
Boolean model of yeast apoptosis as a tool to study yeast and human apoptotic regulations
}

\author{
Laleh Kazemzadeh $^{1,2}$, Marija Cvijovic ${ }^{1,3}$ and Dina Petranovic ${ }^{1 *}$ \\ ${ }^{1}$ Department of Chemical and Biological Engineering, Chalmers University of Technology, Gothenburg, Sweden \\ ${ }^{2}$ Digital Enterprise Research Institute, National University of Ireland, Galway, Ireland \\ ${ }^{3}$ Department of Mathematical Sciences, Chalmers University of Technology and University of Gothenburg, Gothenburg, Sweden
}

Research Topic: From structural to molecular systems biology: experimental and computational approaches to unravel mechanisms of kinase activity regulation in cancer and neurodegeneration

\section{Edited by:}

Matteo Barberis, Humboldt University

Berlin, Germany; Max Planck Institute

for Molecular Genetics, Berlin,

Germany

\section{Reviewed by:}

loannis Xenarios, SIB Swiss Institute of Bioinformatics, Switzerland

Abhishek Garg, Harvard University, USA

\section{*Correspondence}

Marija Cvijovic, Department of Mathematical Sciences, Chalmers University of Technology and

University of Gothenburg, Chalmers tvärgata 3, Göteborg 412 96, Sweden. e-mail:marija.cvijovic@chalmers.se; Dina Petranovic, Department of Chemical and Biological Engineering Chalmers University of Technology, Kemivägen 10, Göteborg SE-412 96, Sweden.

e-mail:dina.petranovic@chalmers.se
Programmed cell death (PCD) is an essential cellular mechanism that is evolutionary conserved, mediated through various pathways and acts by integrating different stimuli. Many diseases such as neurodegenerative diseases and cancers are found to be caused by, or associated with, regulations in the cell death pathways. Yeast Saccharomyces cerevisiae, is a unicellular eukaryotic organism that shares with human cells components and pathways of the PCD and is therefore used as a model organism. Boolean modeling is becoming promising approach to capture qualitative behavior and describe essential properties of such complex networks. Here we present large literature-based and to our knowledge first Boolean model that combines pathways leading to apoptosis (a type of PCD) in yeast. Analysis of the yeast model confirmed experimental findings of anti-apoptotic role of Bir1p and pro-apoptotic role of $\mathrm{Stm} 1 \mathrm{p}$ and revealed activation of the stress protein kinase Hog proposing the maximal level of activation upon heat stress. In addition we extended the yeast model and created an in silico humanized yeast in which human pro- and anti-apoptotic regulators Bcl-2 family and Valosin-contain protein (VCP) are included in the model. We showed that accumulation of Bax in silico humanized yeast shows apoptotic markers and that VCP is essential target of Akt Signaling. The presented Boolean model provides comprehensive description of yeast apoptosis network behavior. Extended model of humanized yeast gives new insights of how complex human disease like neurodegeneration can initially be tested.

Keywords: apoptosis, Boolean modeling, Stm1, Bir1, Hog1, VCP, Bcl-2 family

\section{INTRODUCTION}

Apoptosis is a complex process which is strictly under control of several regulatory networks. Any kind of malfunctioning in these controlling systems due to insufficient or excessive apoptosis signal can potentially lead to threatening diseases such as various types of cancer and neurodegenerative disorders. Therefore keeping this process tightly regulated is important for the cell. Even though apoptosis is often studied in multicellular organisms, the discovery of yeast apoptosis in 1997 (Madeo et al., 1997) attracted the attention of the wide research community (Frohlich et al., 2007; Owsianowski et al., 2008; Madeo et al., 2009; CarmonaGutierrez et al., 2010b). As in other multicellular organisms the apoptosis in yeast is triggered by both internal and external signals. In yeast, the external signals can include acetic acid (Ludovico et al., 2001, 2002), salts, metal ions, ethanol, osmotic stress, heat stress (Madeo et al., 2009), lipids (Aerts et al., 2008; Low et al., 2008; Garbarino et al., 2009), mating pheromone (Zhang et al., 2006), different pharmacological molecules, and drugs (Almeida et al., 2008). Internal signals can include, ammonium, NO, ROS (that can be generated within the cell by mitochondria and the ER, and also induced by $\mathrm{H}_{2} \mathrm{O}_{2}$ addition (Madeo et al., 1999) and other factors), damage (proteins, lipids, nucleic acids) as a consequence of aging and mutations (Mazzoni et al., 2005; Weinberger et al., 2005; Hauptmann et al., 2006), as well as expression of heterologous proteins, such as human pro-apoptotic proteins (Eisenberg et al., 2007). Many proteins residing in the cytoplasm, nucleus, mitochondria, ER, peroxisomes, and lysosomes have been identified as the regulators of apoptosis. For example, proteolysis is one of the main steps that leads to execution of cell death and, a yeast metacaspase Ycalp has been shown to be central for most (but not all) cell death scenarios (Madeo et al., 2002, 2009). Besides degradation of proteins, degradation of nucleic acids is also carried out during apoptotic death and one of the two important caspase-independent mediators is Nuclp (homolog of endonuclease G; Buttner et al., 2007). The second is Aiflp (apoptosis-inducing factor; Wissing et al., 2004) that is together with Nuclp released from the mitochondrion and translocated to the nucleus during the initiation and execution of apoptosis. The regulation of apoptosis in the nucleus, is achieved via pro-apoptotic factor Nma111p (nuclear mediator of apoptosis; Fahrenkrog et al., 2004) a serine protease that cleaves an antiapoptotic factor (inhibitor of apoptosis, IAP) Birlp, which is the only known IAP in yeast, and its anti-apoptotic mechanisms (known to be YCA1-independent) are not well characterized (Walter et al., 2006). To understand how large and complex network of apoptosis process is regulated it should be studied as a whole allowing identification of the properties essential for biological function (Janes et al., 2005). To complement experimental studies 
mathematical models are often use permitting systematic analysis of the network components either individually or jointly (Wolkenhauer, 2002; Stelling, 2004). In Boolean networks (BN) introduced by Kauffman (1969) these assumptions are made based on activation/inhibition effects of one node on another downstream node. The Boolean "on" state (or 1 state or "true" state) can be translate to biological active state of specific species, while "off" state (or 0 state or "false" state) corresponds to inactive state. With simple logical rules (AND, OR, and NOT) it is possible to capture system's behavior in a discrete manner without being dependent on experimental measurements such as molecular concentration or kinetic rates. This type of model implementation is becoming more common in biology (Handorf and Klipp, 2012) and examples include various model organisms and processes, ranging from cell cycle models of simple fission yeast (Davidich and Bornholdt, 2008), to complex dynamic analysis of mammalian cell cycle (Fauré et al., 2006), study of mammalian neurotransmitter signaling pathway (Gupta et al., 2007), investigation of irreversible mammalian apoptosis and stable surviving (Mai and Liu, 2009), and model of apoptosis in human (Schlatter et al., 2009). In each of these studies different extensions of Boolean modeling is implemented giving clear indication of the growing application of logic based modeling in qualitative studies of biological networks where there is not much quantitative data available.

We describe here a model based on Boolean network approach that consists of two parts: in the first part construction and the evaluation of the yeast apoptosis Boolean model is introduced and in the second part we propose the use of established model for study of human apoptotic proteins in yeast. Simplicity of $\mathrm{BN}$ allowed us to construct the model that integrates vast amount of heterogeneous knowledge that currently exists for yeast apoptosis. The major purpose of this study is to understand the emergence of systems properties. Extensive analysis of the state space in combination of different input signals generated series of in silico experiments. Simulating knock out experiments we were able to test the function of specific feedback structures in apoptotic network. The results were compared with the existing experimental data and the model was used to explore several hypotheses in order to better understand certain apoptotic mechanisms and to suggest new strategies for further experimental studies.

\section{RESULTS \\ NETWORK TOPOLOGY}

The constructed yeast apoptosis network contains 73 species and 115 reactions (Table 1 ). Six species do not have successor (Sink/Output) or predecessor (Source/Input; Table 2). In the schematic diagram (Figure 1) species are represented as nodes and reactions as edges. Species include: processes, proteins, and small molecules (metabolites or signals) and Reactions include activation (green arrow) and inhibition (red arrow). Nine nodes depict inputs to the model and are colored blue. Seventeen elements are active in nucleus and are shown in gray, 12 mitochondria species are shown in yellow, and 34 orange boxes represent species residing in cytoplasm. System has only one output node which is called
"Apoptosis" and it is in dark blue. Filled blue circles are used as an "AND" gates between two or more reactions to indicate the necessity of presence of two species for activation or inhibition of a reaction (Figure $\mathbf{1}$ ).

Network is activated via input signals corresponding to the following species: Acetic Acid, Heat, $\mathrm{H}_{2} \mathrm{O}_{2}$, Adozelesin, $\mathrm{Mg}^{2+}, \mathrm{Cu}^{2+}$, Salt $(\mathrm{NaCl})$, mating, and osmotic stress.

\section{MODEL PROPERTIES}

Like many molecular mechanisms in living cells, apoptosis can be approximated as an outcome of sequential regulation steps which do not occur all at the same time. As a result, upon induction, the state of the cell changes with passing time. In order to capture this feature, each reaction was assigned activation time scale implicating occurrences of different scenarios in sequential order. By introducing time delays to the logical function, it is possible to describe dynamic behavior of the given process using logical networks (Thomas and D'Ari, 1990).

For this study we used Cell Net Analyser (CNA; Klamt et al., 2006) which provides the function to capture signal propagation in a time series to get a snapshot of the network and discrimination of signaling events. This approach has been successfully used to model human apoptotic network revealing new feedback loops (Schlatter et al., 2009). To describe dynamic behavior of the yeast apoptosis five timescales $t=[0,2,4,5,6]$ are assigned. These timescales are constants and indicate at which timescale each node gets activated or inhibited. Simulation of the network at $t=x$ comprises of all interactions with timescale $t \leq x$ but not interactions with $t \geq x$. This gives the possibility of separation of different functional groups such as different signaling pathways or feedback loops. It should be noted that specified timescales do not refer to real time but are only indicators of sequential regulatory steps (difference between timescale $t=0$ and $t=2$ is equal to difference between timescales $t=2$ and $t=4$ or $t=4$ and $t=5$ or between $t=5$ and $t=6$ ). Timescales are not assigned based on speed of reactions (how fast or slow reactions are), they are assigned based on the sequence of events. To allow flexibility in our model and facilitate changes and insertions of new events timescales 1 and 3 are reserved and not used in the current network structure. First timescale $t=0$ is assigned to genes which are already present and constantly active in cell (27 reactions). Values for all input arcs to stimuli species are set to 1 at second timescale $t=2$ (53 reactions). Further interactions are activated at time $t=4$ (17 reactions). Although yeast apoptosis does not contain any feedback loops time point $t=5$ is reserved for feedback loops which get activated in response to osmotic and heats shock and will lead to apoptosis. Considering impact of feedback loops on system behavior it is reasonable to assign them a separate timescale. Finally interaction occurring at the very end are assigned timescale $t=6$ (18 reactions). Details on each reaction, their time points and species involved in reactions are in Table 3.

The model simulated the induction of apoptosis both in the independent mode (assessing each individual stimulus separately) as well as in the additive mode, where the activation of all inputs were set at the same time (Table 4). 
Table 1 | List of species in yeast Boolean model.

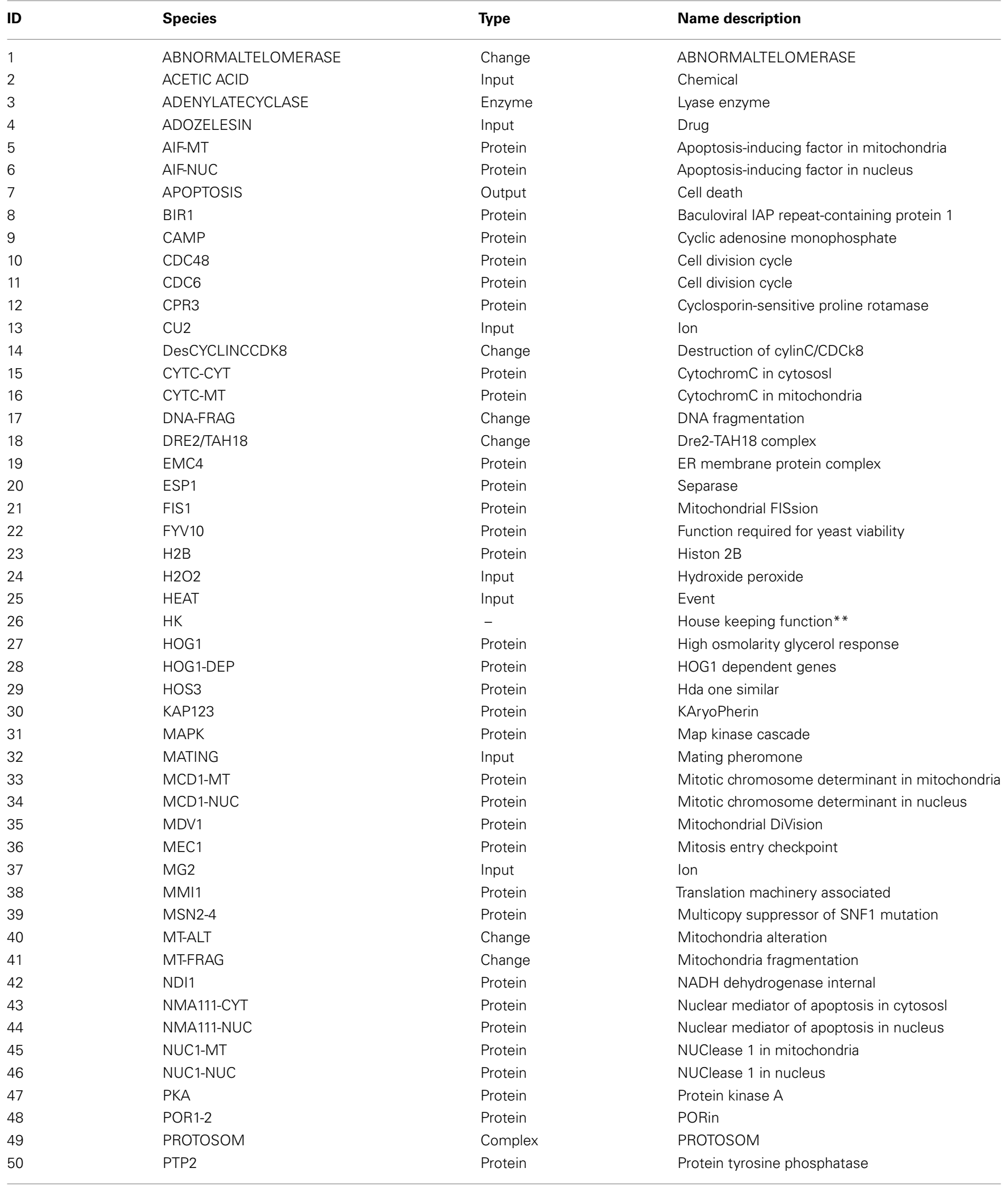


Table 1 | Continued

\begin{tabular}{|c|c|c|c|}
\hline ID & Species & Type & Name description \\
\hline 51 & PTP3 & Protein & Protein tyrosine phosphatase \\
\hline 52 & RAS2 & Protein & Homologous to RAS proto-oncogene \\
\hline 53 & RedActinDyn & Change & Reduced actin dynamic \\
\hline 54 & RLM1 & Protein & Resistance to lethality of MKK1P386 overexpression \\
\hline 55 & ROS-CYT & Molecule & Reactive oxygen species in cytososl \\
\hline 56 & ROS-MT & Molecule & Reactive oxygen species in mitochondria \\
\hline 57 & RPD3 & Protein & Reduced potassium dependency \\
\hline 58 & SALT & Input & - \\
\hline 59 & SDP1 & Protein & Stress-inducible dual specificity phosphatase \\
\hline 60 & SLT2 & Protein & SYNtaxin (SYN8) \\
\hline 61 & SNO1 & Protein & SNZ proximal open reading frame \\
\hline 62 & SOD1 & Protein & Superoxide dismutase \\
\hline 63 & SOD2 & Protein & Superoxide dismutase \\
\hline 64 & $\mathrm{SRO7}$ & Protein & Suppressor of rho3 \\
\hline 65 & STE20-CYT & Protein & Sterile in cytosol \\
\hline 66 & STE20-NUC & Protein & Sterile in nucleus \\
\hline 67 & STM1-CYT & Protein & Translation initiation factor (TIF3) in cytososl \\
\hline 68 & STM1-NUC & Protein & Translation initiation factor (TIF3) in nucleus \\
\hline 69 & STRESS & Input & Event \\
\hline 70 & SVF1 & Protein & SurVival factor \\
\hline 71 & TAT-D & Protein & $3^{\prime} \rightarrow 5^{\prime}$ exonuclease and endonuclease \\
\hline 72 & TOR1 & Protein & Target of rapamycin \\
\hline 73 & YCA1 & Protein & MetaCAspase \\
\hline
\end{tabular}

Table includes name, type, and description of each species involved in yeast apoptosis.

* Species refers to all types of nodes that are depicted in the network map and are color coded based on their location or activity in cell.

**Housekeeping function refers to those genes which are present and give snapshot of state of the cell before applying any kind of treatment.

Table 2 | Summary of the interactions without successor (Sink/Output) and predecessor (Source/Input).

\begin{tabular}{lll}
\hline Species & Type of connection & Number of connections \\
\hline Ros-MT & Sink/output & 1 \\
MCD1-NUC & Sink/output & 1 \\
H2B & Sink/output & 5 \\
CAMP & Sink/output & 2 \\
RedActinDyn & Source/input & 3 \\
AbnormalTelomer & Source/input & 1 \\
HK & Source/input & 18 \\
\hline
\end{tabular}

Table contains species without successor (there are no edges coming out of these nodes) and predecessor (there are no edges going to these nodes).

\section{PREDICTIONS WITH THE CONTINUOUS MODEL}

Based on qualitative knowledge we have constructed a discrete Boolean model of yeast apoptosis. While it can capture its essential behavior, the question remained how this model can be used to predict the qualitative behavior of the system. In order to address this question we expanded the model by transforming the discrete Boolean model into a continuous model using Odefy (Wittmann et al., 2009; Krumsiek et al., 2010; see Materials and Methods). In order to test the continuous model and its predictive capacity, we performed three independent case-studies: (i) induction of apoptosis by activation of Hog1p by heat stress, (ii) inhibition by Birlp of acetic acid-induced apoptosis, and (iii) induction of apoptosis with $\mathrm{H}_{2} \mathrm{O}_{2}$ by activation of Stmlp. We show here that the behavior that emerges from specific interactions in the model is in agreement with published experimental data.

\section{ACTIVATION OF HEAT STRESS-INDUCED APOPTOSIS WITH Hog1p}

We simulated the activation of the mitogen-activated protein kinase that is a key component of the HOG pathway (Albertyn and Hohmann, 1994; Van Wuytswinkel et al., 2000; Hohmann, 2002; de Nadal et al., 2004), which is an osmoregulatory signal transduction cascade (Hohmann, 2009). Upon stress, Hoglp (encoded by HOG1/YLR113W) regulates the expression of almost 600 genes by phosphorylating several different transcription factors (Hohmann, 2002; Westfall et al., 2004). The activity and nuclear localization of Hog1p is regulated by its phosphorylation state, and that in turn is regulated by the kinase MAPKK Pbs2p and the phosphatases Ptc1p, Ptc2p, Ptc3p, Ptp2p, and Ptp3p (Brewster et al., 1993; Ferrigno et al., 1998; Warmka et al., 2001; Young et al., 2002). Besides the induction by osmotic stress, the HOG pathway can be induced by heat stress, via a Sholp-dependent sensory mechanism (Winkler et al., 2002), thus we used heat stress as an input to activate the HOG pathway and simulate the cell death response. Heat stress activates Slt2 which then activates Rlm1. Consequently Rlm1 triggers expression of Slt2 and PTP2 forming a feedback loop. On the other hand heat shock inhibits activity 


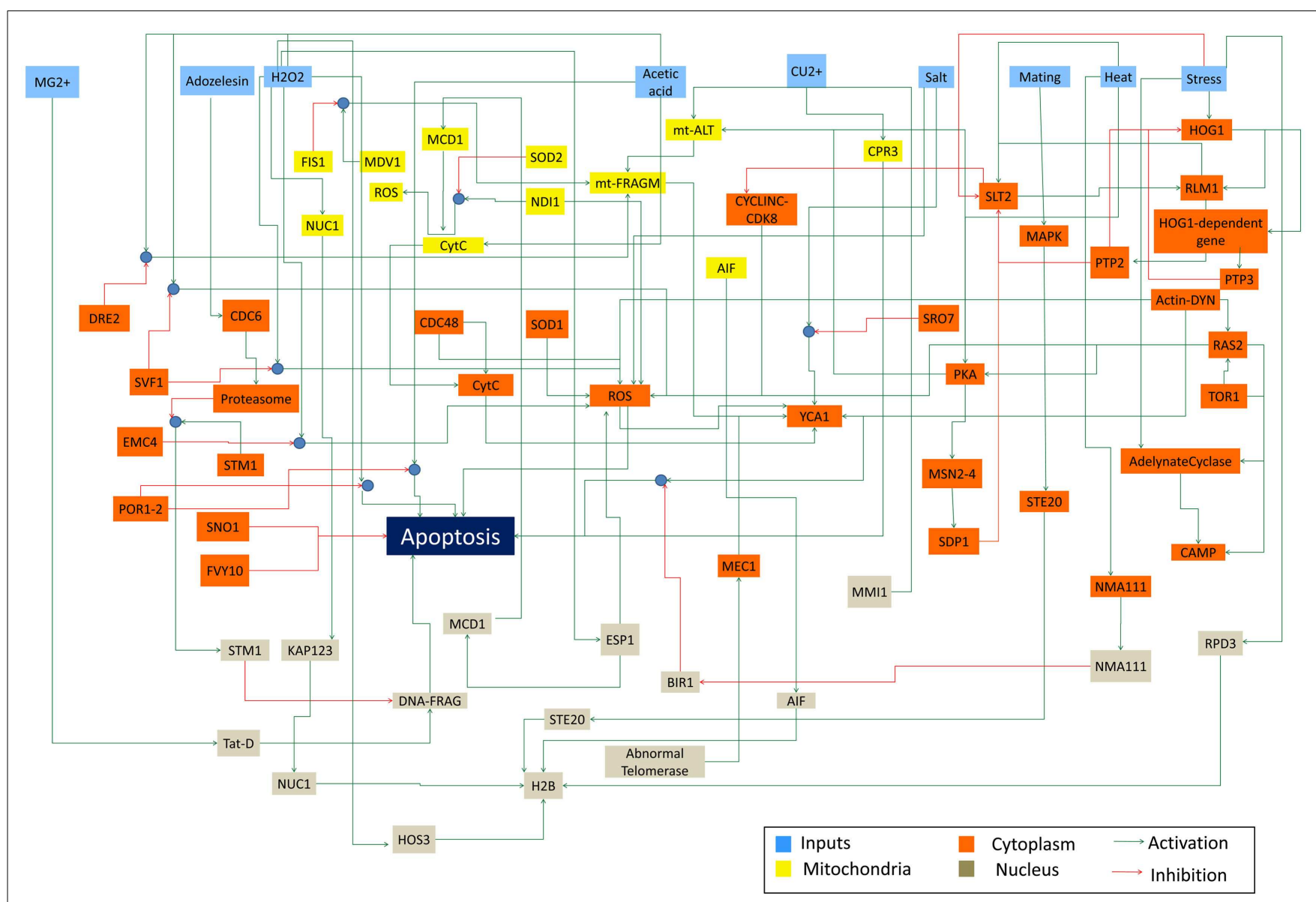

FIGURE 1 | Schematic representation of Yeast Apoptosis network. Blue boxes depict input nodes, yellow nodes are placed in mitochondria, orange nodes reside in cytosol and grey nodes belong to nucleus. Green arrows show activation effect and red arrows show inhibition effect. Blue circles depict "AND" gate.

of PKA leading to release the inhibition effect of PKA on MSN24. MSN2-4 activates Sdp1 which then along with PTP2 inhibit Slt2. We started by setting all other stimuli to zero and then by transforming the Boolean apoptosis model into Hill Cube continuous model we performed the simulations. Steady states predicted from continuous Hill Cube (Figure 2) and synchronous Boolean (Figure 3) model are in perfect agreement with each other for all nodes apart from the nodes corresponding to the feedback loops in response to heat and osmotic stress. Feedback loop in heat activated pathway includes activation of Rlm 1 by Slt 2 and expression of Slt 2 by Rlm1. Osmotic shock induced Hog1 activates Rlm1 to regulate Slt2 which was inhibited by osmotic shock and PTP2. Unknown Hog1 dependent transcription factor triggers transcription of PTP3 and PTP2 resulting in dephosphorylation of Hog1 phosphotyrosine which inhibits Hogl activities. All nodes in our model were connected to activation of apoptosis via the heat shock pathway and were indeed activated in our simulation and are colored in red; other pathways are colored in blue indicating that they are not activated during the simulation (Figure 2). Model predicts that upon heat induction, concentration of Hoglp changes trough time never reaching its maximum level of concentration and as an intensity of stimulus decreases level of Hoglp also decreases. Our simulations also proposed that the maximal level of activation of Hog 1 p during heat stress is $70 \%$ of the total activation and as the heat stimulus continues over time its activity decreases and reaches a plateau at $40 \%$ of total activation (Figure 4 ).

This model prediction is in agreement with an experimental measurement performed by Winkler et al. (2002) which shows the induced activity of Hoglp upon heat induction (Figure 4). The difference between the maximal activity values and the lag phase of the activation is due to the fact that we did not use any quantitative data as input to the model.

\section{INHIBITION OF ACETIC ACID-INDUCED APOPTOSIS WITH Bir1p}

Even though there are several genes and pathways that are involved in yeast pro-apoptotic response, there are only few anti-apoptotic genes. For many years Bir1p (encoded by BIR1/YJR089W) was thought to be the only apoptotic inhibitor in yeast, but recently a TSC22-protein family was found and two of the proteins with TSC22-motif have been shown to have anti-apoptotic roles Snolp and Fvy10p (Khoury et al., 2008). Birl belongs to the IAP family and phylogenetic analysis (Uren et al., 1998) showed similarity to Schizosaccharomyces pombe BIR1, human survivin, and Caenorhabditis elegance BIR-1 and BIR-2 proteins. Yeast's 
Table 3 | List of logical interactions in yeast Boolean model.

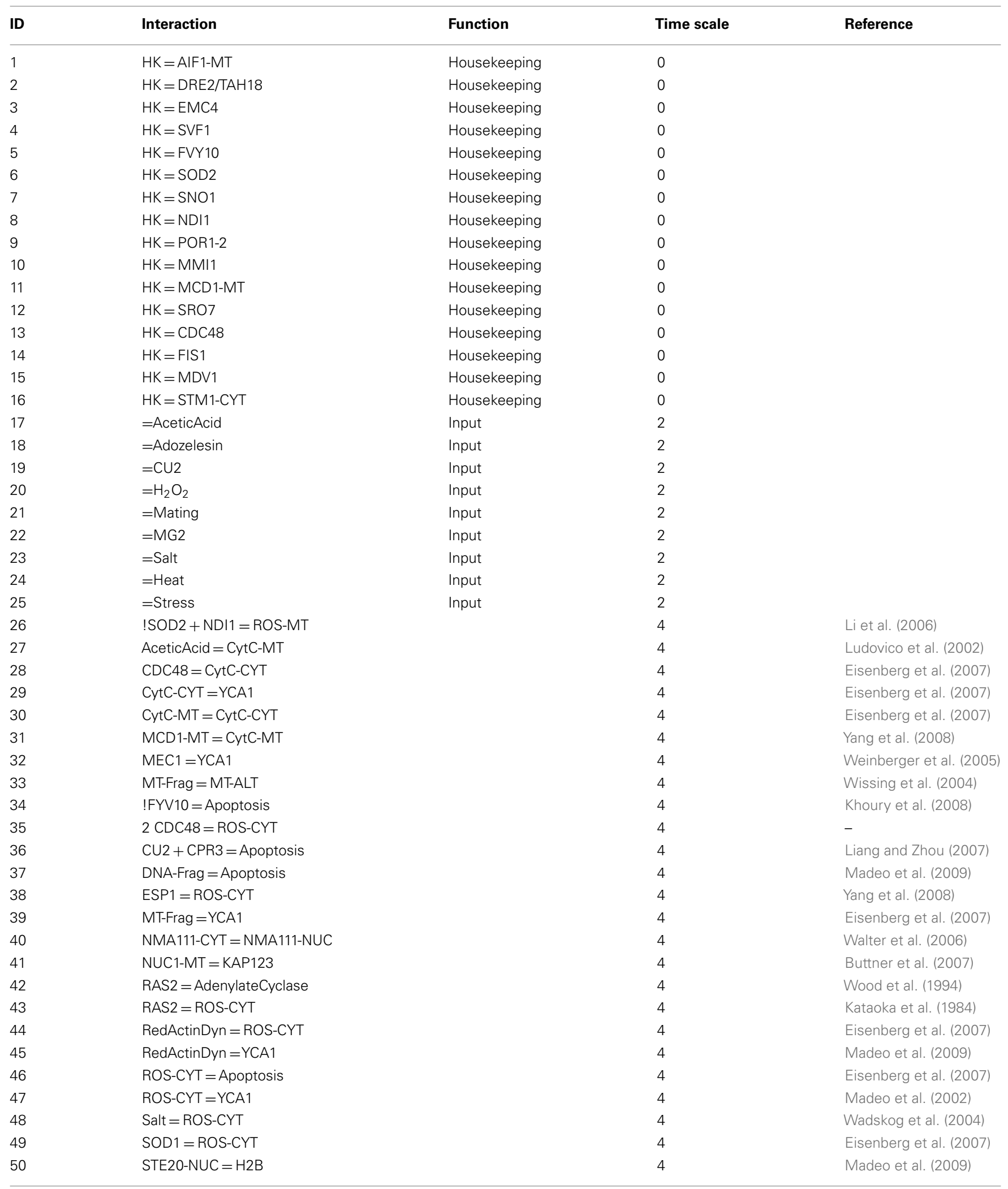


Table 3 | Continued

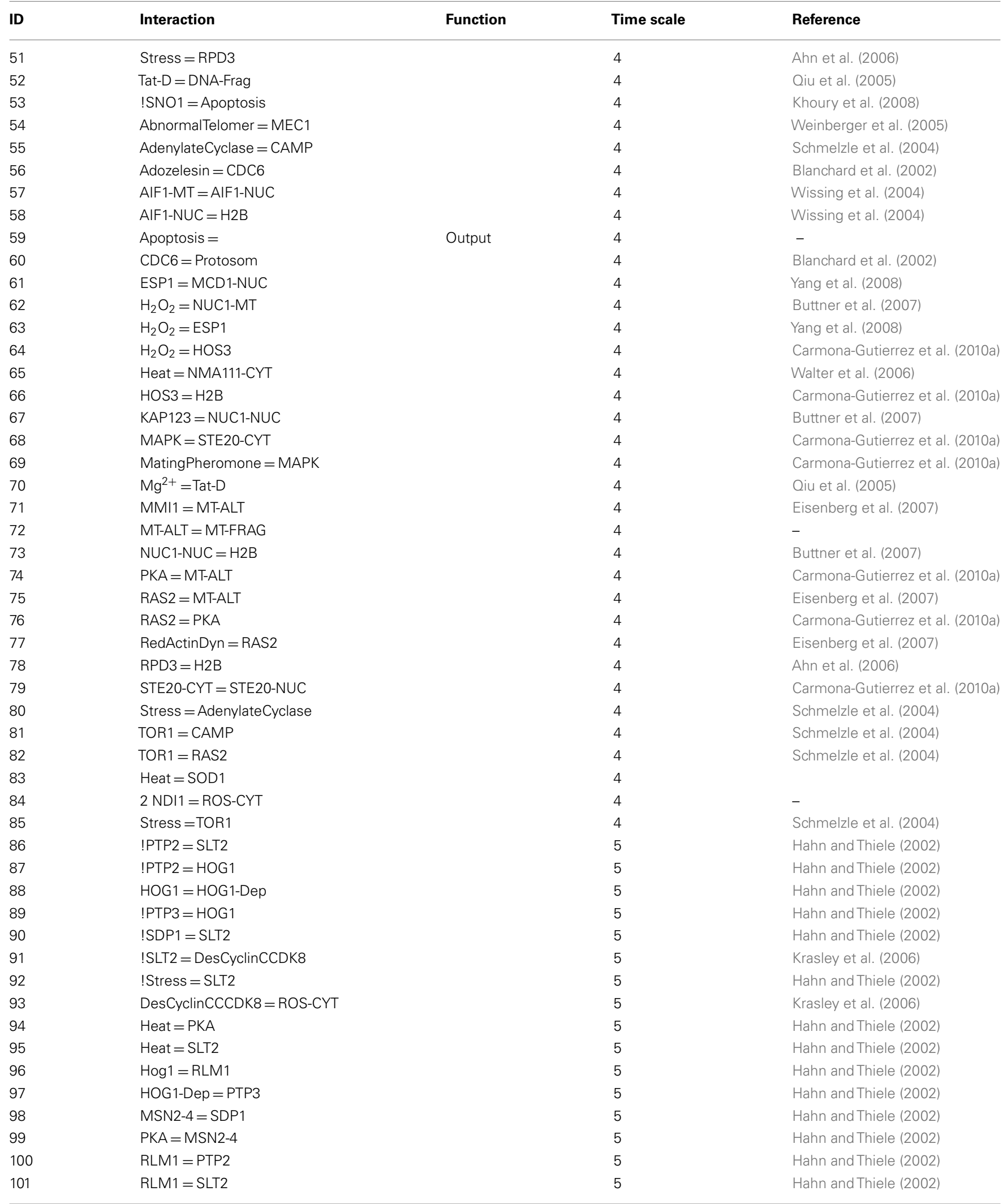


Table 3 | Continued

\begin{tabular}{|c|c|c|c|c|}
\hline ID & Interaction & Function & Time scale & Reference \\
\hline 102 & $\mathrm{SLT} 2=\mathrm{RLM} 1$ & & 5 & Hahn and Thiele (2002) \\
\hline 103 & Stress = HOG1 & & 5 & Hahn and Thiele (2002) \\
\hline 104 & !SRO7 + Salt =YCA1 & & 6 & Wadskog et al. (2004) \\
\hline 105 & !STM1-NUC = DNA-Frag & & 6 & Ligr et al. (2001) \\
\hline 106 & AceticAcid + !SVF1 = ROS-CYT & & 6 & Vander Heiden et al. (2002) \\
\hline 107 & $\mathrm{H}_{2} \mathrm{O}_{2}+$ !EMC4 $=\mathrm{ROS}-\mathrm{CYT}$ & & 6 & Ring et al. (2008) \\
\hline 108 & $\mathrm{H}_{2} \mathrm{O}_{2}+$ !SVF1 $=\mathrm{ROS}-\mathrm{CYT}$ & & 6 & Vander Heiden et al. (2002) \\
\hline 109 & !FIS1 + MDV1 = MT-Frag & & 6 & Eisenberg et al. (2007) \\
\hline 110 & !POR1-2 + AceticAcid = Apoptosis & & 6 & Pereira et al. (2007) \\
\hline 111 & !POR1-2 $+\mathrm{H}_{2} \mathrm{O}_{2}=$ Apoptosis & & 6 & Pereira et al. (2007) \\
\hline 112 & $\mathrm{H}_{2} \mathrm{O}_{2}+$ !DRE2/TAH18 = MT-Frag & & 6 & Vernis et al. (2009) \\
\hline 113 & YCA1 + !BIR1 = Apoptosis & & 6 & Walter et al. (2006) \\
\hline 114 & !NMA111-NUC = BIR1 & & 6 & Walter et al. (2006) \\
\hline 115 & STM1-CYT + !Protosom = STM1-NUC & & 6 & Ligr et al. (2001) \\
\hline
\end{tabular}

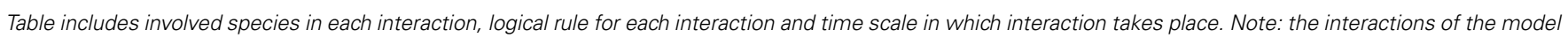

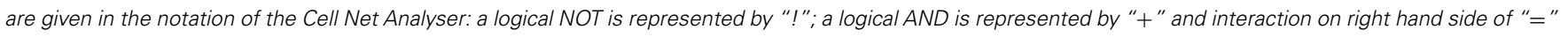
gives the value of node on left hand side or equation.

Table 4 | Predicted states of relevant species at steady state.

\begin{tabular}{lllll}
\hline Species & $\boldsymbol{t}=\mathbf{0}$ & $\boldsymbol{t}=\mathbf{4}$ & $\boldsymbol{t}=\mathbf{5}$ & $\boldsymbol{t}=\mathbf{6}$ \\
\hline Acetic acid & 1 & 1 & 1 & 1 \\
Adozelesin & 1 & 1 & 1 & 1 \\
AIF1-MT & 1 & 1 & 1 & 1 \\
AIF1-NUC & 0 & 1 & 1 & 1 \\
Apoptosis & 0 & 0 & 0 & 1 \\
BIR1 & 0 & 0 & 0 & 0 \\
DNA-FRAG & 0 & 1 & 1 & 1 \\
H2O2 & 1 & 1 & 1 & 1 \\
Heat & 1 & 1 & 1 & 1 \\
NMA111-NUC & 0 & 1 & 1 & 1 \\
ROS-CYT & 0 & 1 & 1 & 1 \\
STE20-CYT & 0 & 1 & 1 & 1 \\
STM1-CYT & 1 & 1 & 1 & 0 \\
STM1-NUC & 0 & 0 & 0 & 1 \\
YCA1 & 0 & 1 & 1 & 1 \\
& & & &
\end{tabular}

Table includes time scale scenario upon induction of all stimuli. T stands for different time steps. As an example if Acetic Acid is inducted to the cell at time point 0 it is expected to cause DNA Fragmentation at next time step and eventually ends to apoptosis in last step.

Birlp has been previously intensively studied in chromosome segregation (as a component of the Aurora kinase complex (chromosome passenger complex, CPC; Ruchaud et al., 2007) but recently studies of its role as a negative regulator of apoptosis have gained momentum. It has been shown that Birlp is a target for degradation by Nma111p (Owsianowski et al., 2008), an apoptotic serine protease and yeast cells lacking Bir1 are more sensitive to apoptosis, while overexpression of Birl reduces apoptosis (Walter et al., 2006).
When building the model we assumed that addition of acetic acid induces cytochrome $c$ release from mitochondria and its translocation to the cytosol. Another assumption is that the execution of apoptosis is Ycalp-dependent (encoded by YCA1/MCA1/YOR197W) in this context and that the activation of the caspase is downstream from the cytochrome $c$ translocation (Ludovico et al., 2001, 2002). Thus, taking these assumptions into account acetic acid was used as an inducer in the simulations with direct induction of apoptosis. The model confirmed experimental evidence that Birl is indeed apoptosis inhibitor in yeast (Walter et al., 2006) and as acetic acid is applied as a pulse stimulus (that is then decreasing over time) and cytochrome $c$ present in the cytosol increases, the decrease of Birlp due to Nma111p degradation promotes apoptosis that then reaches the maximum (Figure 5A). We then performed an artificial and biologically irrelevant simulation: after apoptosis has occurred and no more inducer was present (acetic acid is not added again and the previously added amount has been "used") the eventual theoretical accumulation of Birlp did inhibit apoptosis and revert it to zero. Obviously this scenario is biologically implausible, since once the cell has undergone apoptosis it cannot be revived, but has a purpose to show mathematical correctness of the developed model.

Additionally, we have converted discreet apoptosis model to continuous model with constant presence of Bir1 in order to test if this type of model would predict the same outcome as the experimental approach in which Birlp is overexpressed and provides protection from induction of apoptosis. The continuous model was created taking into account translocation effect of each element from one compartment to another. This effect has been applied to translocation of Nucl from mitochondria to nucleus, MCD1 from nucleus to mitochondria, Stm1 from cytosol to nucleus Aif1 (from mitochondria to nucleus, NMA111 from cytosol to nucleus, Ste20 from cytosol to nucleus, and cytochrome 


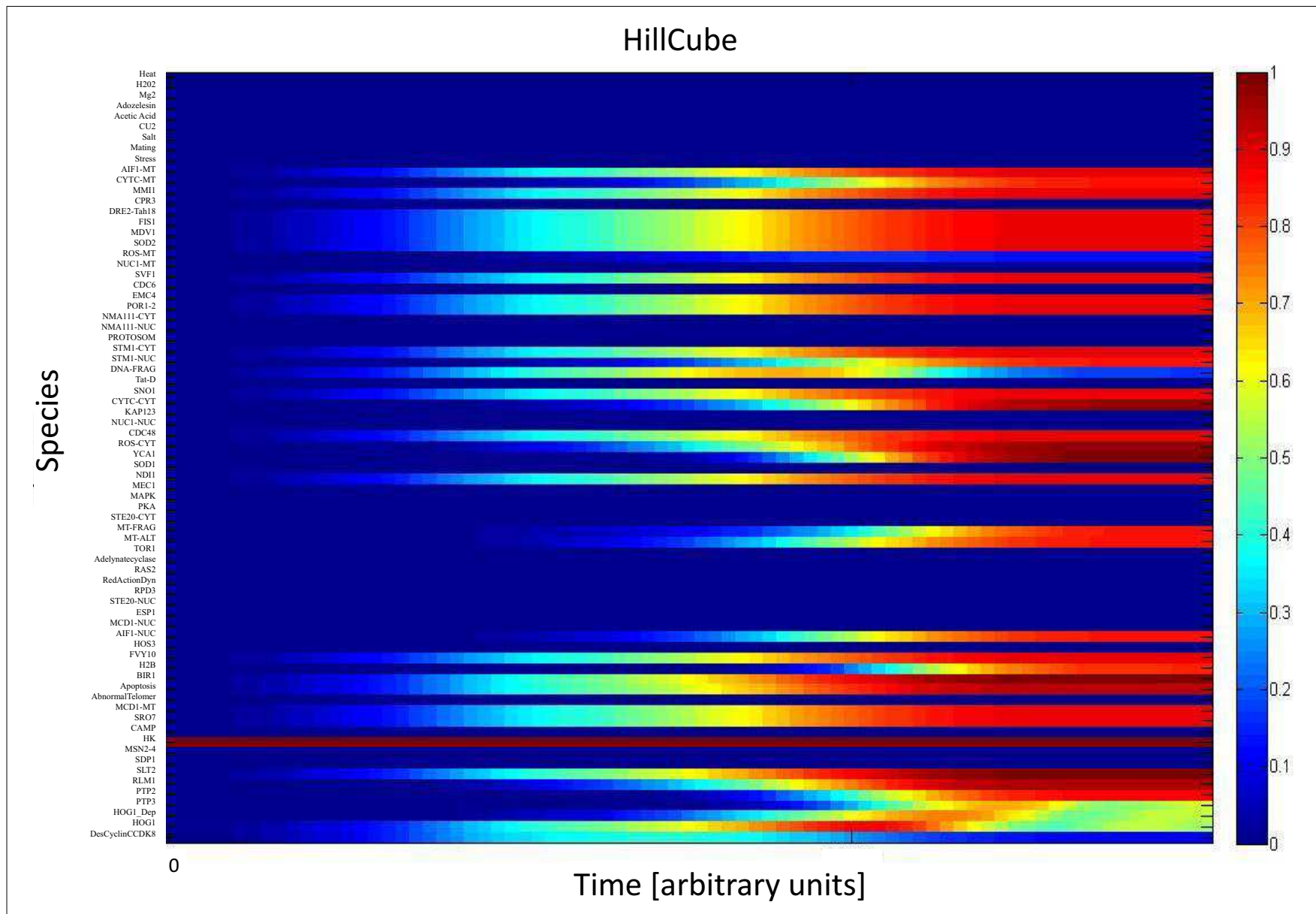

FIGURE 2 | Continuous Hill Cube transformation. All species in apoptosis network are mapped on vertical axis. Dark blue color indicates those nodes that are not activated (value $=0$ ) while dark red refers to nodes which are completely activated (value $=1$ ). Each color in between indicates the level of activation between 0 and 1 .

c from mitochondria to cytosol. Initially, in this model, translocation of each species is modeled as one node in order to represent the re-localization which introduced self-regulatory loops to the system which is impossible since to our knowledge yeast apoptosis regulatory network does not have such loops. One solution implies intuitive approach in defining single variable for single species to mimic the effect of transferring from one compartment to another. Technically this was solved by introducing two variables for a single species representing both compartments they can belong to. This approach, also, confirmed experimental finding (Walter et al., 2006) that constant presence of Bir1, inhibits apoptosis, validating Bir1 anti-apoptotic role (Figure 5B).

\section{ACTIVATION OF $\mathrm{H}_{2} \mathrm{O}_{2}$-INDUCED APOPTOSIS WITH Stm1p}

It has been shown that degradation of short-lived pro-apoptotic proteins via proteasomal - ubiquitine pathway plays important role in mammalian apoptosis (Drexler, 1997). One of the yeast proteasomal substrates - Stm1 (YLR150W) was identified in the study by Ligr et al. (2001) as an activator of the cell death process. Conserved orthologs of Stm 1 are detected in several species (highly conserved ortholog in Schizosaccharomyces pombe and a putative ortholog in Drosophila melanogaster (Nelson et al., 2000) suggesting that regulation of apoptosis via this protein can be evolutionary conserved process.

We have in silico tested two scenarios confirming the role of Stm1 in apoptosis (Ligr et al., 2001). Accumulation of Stm1 will induce apoptotic behavior followed by DNA fragmentation which is a known marker of cell death, while the Stm1 knock out will promote survival and consequently deterring DNA fragmentation (Figures 6A,B).

\section{IN SILICO HUMANIZED YEAST APOPTOSIS}

$S$. cerevisiae is a model organism that has conserved genes, proteins and pathways that are similar to the ones in the human cells. This allows for using yeast as a host in which human genes can be expressed, so called "humanized yeast" (Winderickx et al., 2008) and subsequently the physiological roles and molecular mechanisms can be studied. In order to test if our system could be used in a similar way (in silico humanized yeast) and provide reliable simulations and predictions, we inserted three human genes with complete downstream pathways into our initial yeast apoptosis 


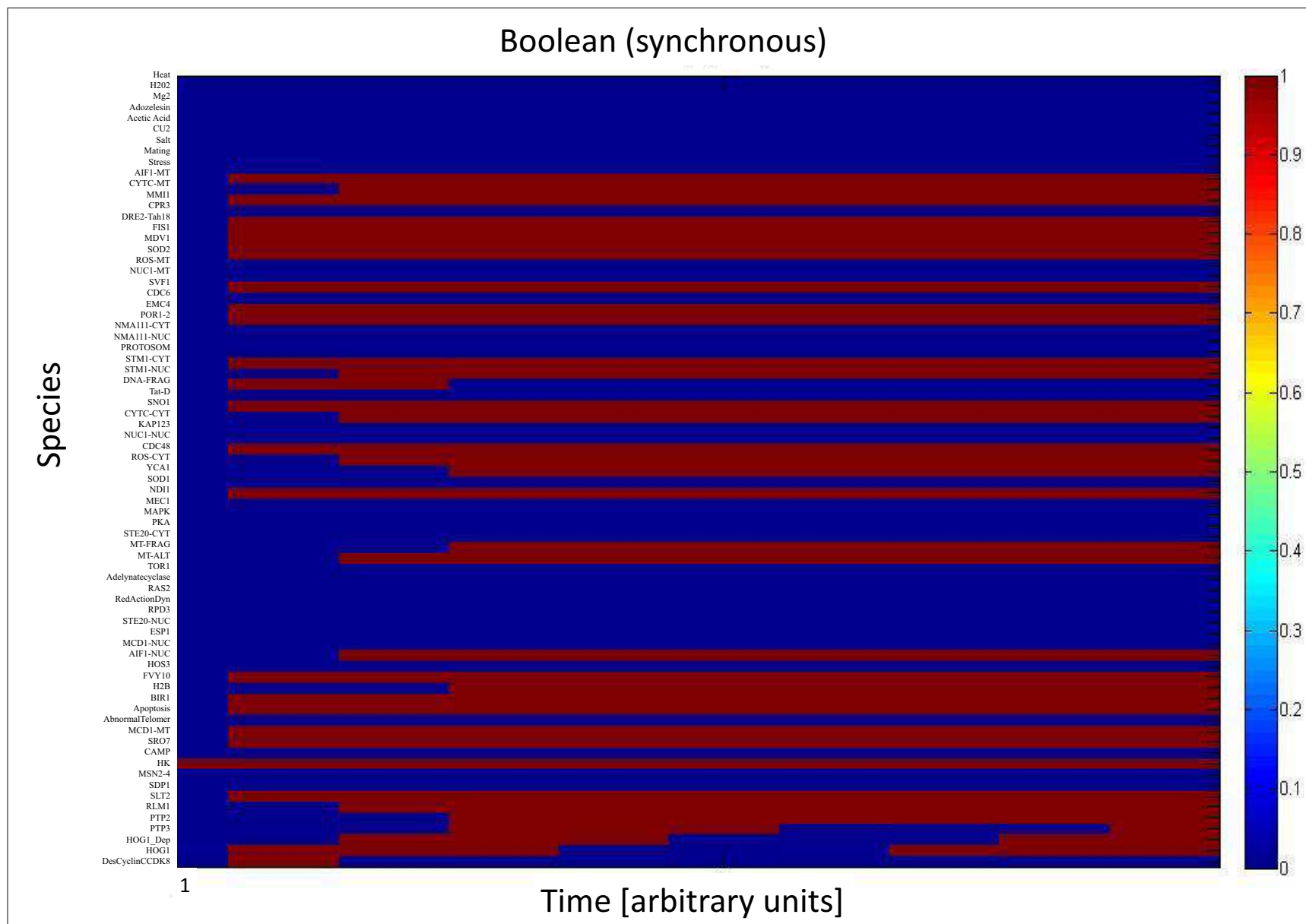

FIGURE 3 | Synchronous Boolean model. All species in apoptosis network are mapped on vertical axis. Dark blue indicates those nodes that are not activated (value $=0$ ) while dark red refers to nodes which are totally activated (value $=1$ ).

Boolean model. Yeast apoptotic network was "humanized" by in silico insertion of genes belonging to $\mathrm{Bcl}-2$ protein family and Valosin-contain protein (VCP) - distinctive representatives of human apoptosis (Figure 7).

\section{INSERTION OF Bcl-2 FAMILY PROTEINS: ANTI-APOPTOTIC BcI-XL AND PRO-APOTOTIC BAX}

This protein family consists of apoptotic agonist and anti-agonist proteins. Among members of Bcl-2 family Bax as an apoptotic inducer and $\mathrm{Bcl}-\mathrm{xL}$ as an anti-apoptotic factor were included in our extended model. Bax is located in cytosol in mammalian cells and has vital role in mitochondria morphogenesis. Heterologously expressed Bax causes growth arrest and rapid cell death in S. cerevisiae (Sato et al., 1994; Greenhalf et al., 1996). Also expression of Bax has been linked to the release of cytochrome $c$ from mitochondria (Manon et al., 1997). Yeast cells with Bax expression accumulate ROS and show other apoptotic hallmarks such as DNA fragmentation (Madeo et al., 1999).

Since members of this protein family were previously successfully expressed in yeast, we first validate the "humanized" yeast model by inserting Bcl-xL, Bax, Bad, and Bcl-2 genes (Sato et al., 1994; Greenhalf et al., 1996; Madeo et al., 1999). As an input signal human Akt signaling was used (Akt Signaling), as an extrinsic regulatory switch (since this signaling cascade is not present in yeast). The Akt Serine/Threonine-kinase promotes cell survival by phosphorylating the pro-apoptotic protein BAD (member of the $\mathrm{Bcl}-2$ family), which is the cause of dissociation of BAD from the $\mathrm{Bcl}-2 / \mathrm{Bcl}-\mathrm{X}$ complex, and promotion of cell survival (Datta et al., 1997). Besides cell survival, Akt signaling is related to the cell cycle, metabolism, and angiogenesis and therefore a target for anticancer drug development (Falasca, 2010; Hers et al., 2011).

Upon activation of Akt Signaling at the first time step $(t=0$, node value Akt Signaling $=1), \mathrm{Bcl}-2$ and $\mathrm{Bcl}-\mathrm{xL}$ are activated in the following step $(t=4$, node value $\mathrm{Bcl}-\mathrm{xL}=1$ and $\mathrm{Bcl}-2=1)$ and remain active throughout the simulation and as the inhibitors of apoptosis promote the survival (node value Apoptosis =0; Table 5). Simulation results suggest the anti-apoptotic role of two $\mathrm{Bcl}-2$ family members: $\mathrm{Bcl}-2$ and $\mathrm{Bcl}-\mathrm{xL}$ which is consistent with experimental findings (Kharbanda et al., 1997).

\section{DUAL FUNCTIONALITY OF VCP IN SURVIVAL AND APOPTOSIS}

The evolutionary conserved Valosin-containing protein (VCP) is a mammalian ortholog of yeast $\mathrm{Cdc} 48$, which is the first apoptotic mediator found in S. cereivisae (Braun and Zischka, 2008). VCP is 
the member AAA-ATPase family which is ubiquitously expressed (Braun and Zischka, 2008). It consists of four domains: N-terminal domain, two ATPase domains D1 and D2 and C-terminal domain (Wang et al., 2004). The major ATPase activity of VCP is carried

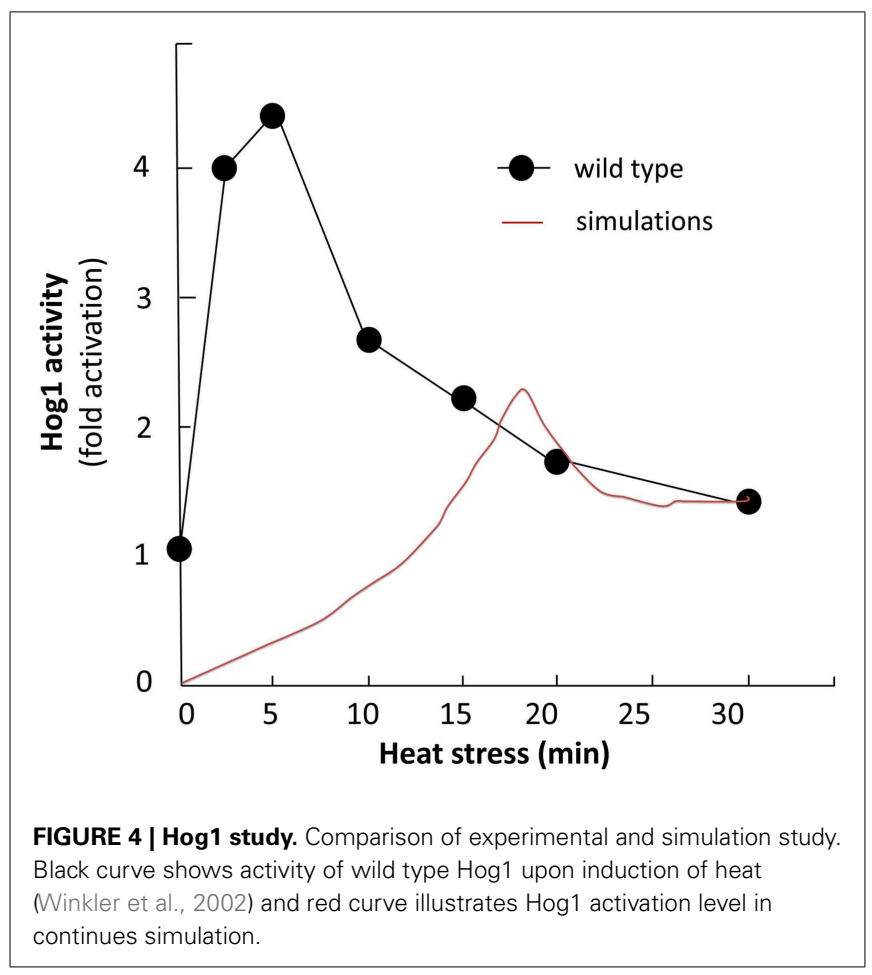

on by D2 domain (Wang et al., 2004; Song et al., 2003). VCP is involved in different cellular process such as protein degradation, membrane fusion and chaperone activity (Braun and Zischka, 2008). Role of VCP/Cdc48 in fluctuating number of death cell in various types of disease naming cancer and protein deposition diseases is not well understood. Increase in expression of VCP is correlated to the development of cancer and metastasis therefore detecting the level of VCP expression is proposed as cancer progression marker. Also VCP is known as detector of aggregated proteins causing neurodegenerative disease such as Parkinson and Alzheimer (Hirabayashi et al., 2001; Mizuno et al., 2003; Ishigaki et al., 2004). It has been observed that yeast cell carrying mutation of Cdc48 is showing morphological markers of apoptosis (Madeo et al., 1997) and that depletion of this protein has apoptotic effect in other organisms as well (Imamuraab et al., 2003).

It had been shown that VCP can both promote or inhibit apoptosis (Braun and Zischka, 2008). Deletion of VCP triggers ER stress which is followed by unfolded protein response and cell death via ER-associated degradation (ERAD) pathway - a highly conserved pathway between mammalian and yeast cells (Braun and Zischka, 2008). Wild type VCP has pro-apoptotic role in the cells undergoing apoptosis upon ER stress which in turn triggers ER-associated apoptotic pathway. Finally VCP can trigger survival pathway in response to $\mathrm{NFkB}$ which is a pro-survival molecule in cells with over expressed level of VCP/Cdc48(Braun and Zischka, 2008).

The dual functionality of VCP in survival and apoptosis mechanism makes it an attractive candidate to be inserted in initial yeast apoptosis Boolean network. Since VCP includes expression of a handful of heterologous proteins, its in vivo insertion to yeast plasmid would be difficult to perform and thus represents a
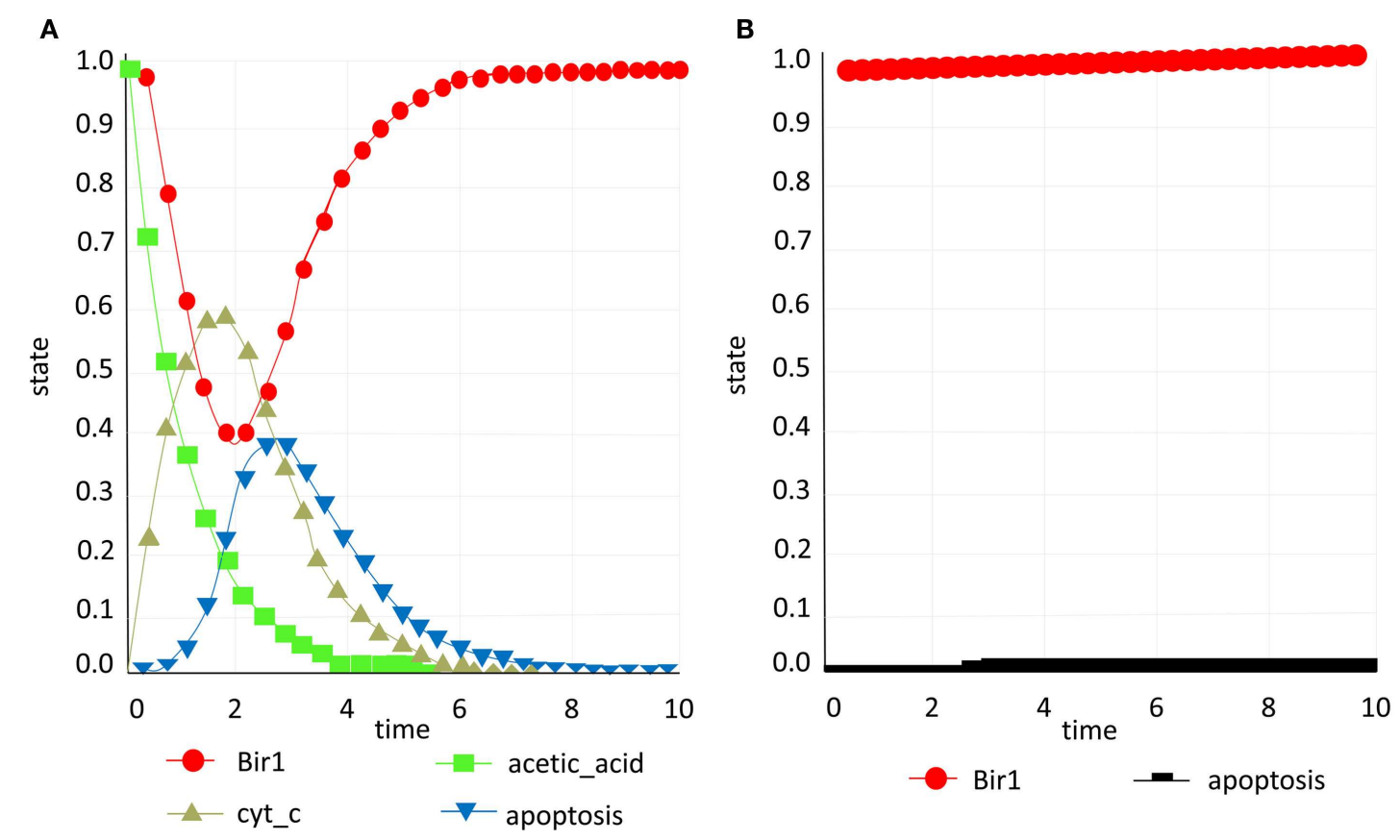

FIGURE 5 | Bir1 study. (A) Acetic acid (green) is applied as a pulse stimulus (that is then decreasing over time) and cytochrome $c$ (yellow) present in the cytosol increases, the decrease of Bir1p (red) promotes apoptosis (B) Constant presence of Bir1p (red) inhibits apoptosis (black), validating Bir1 anti-apoptotic role. 

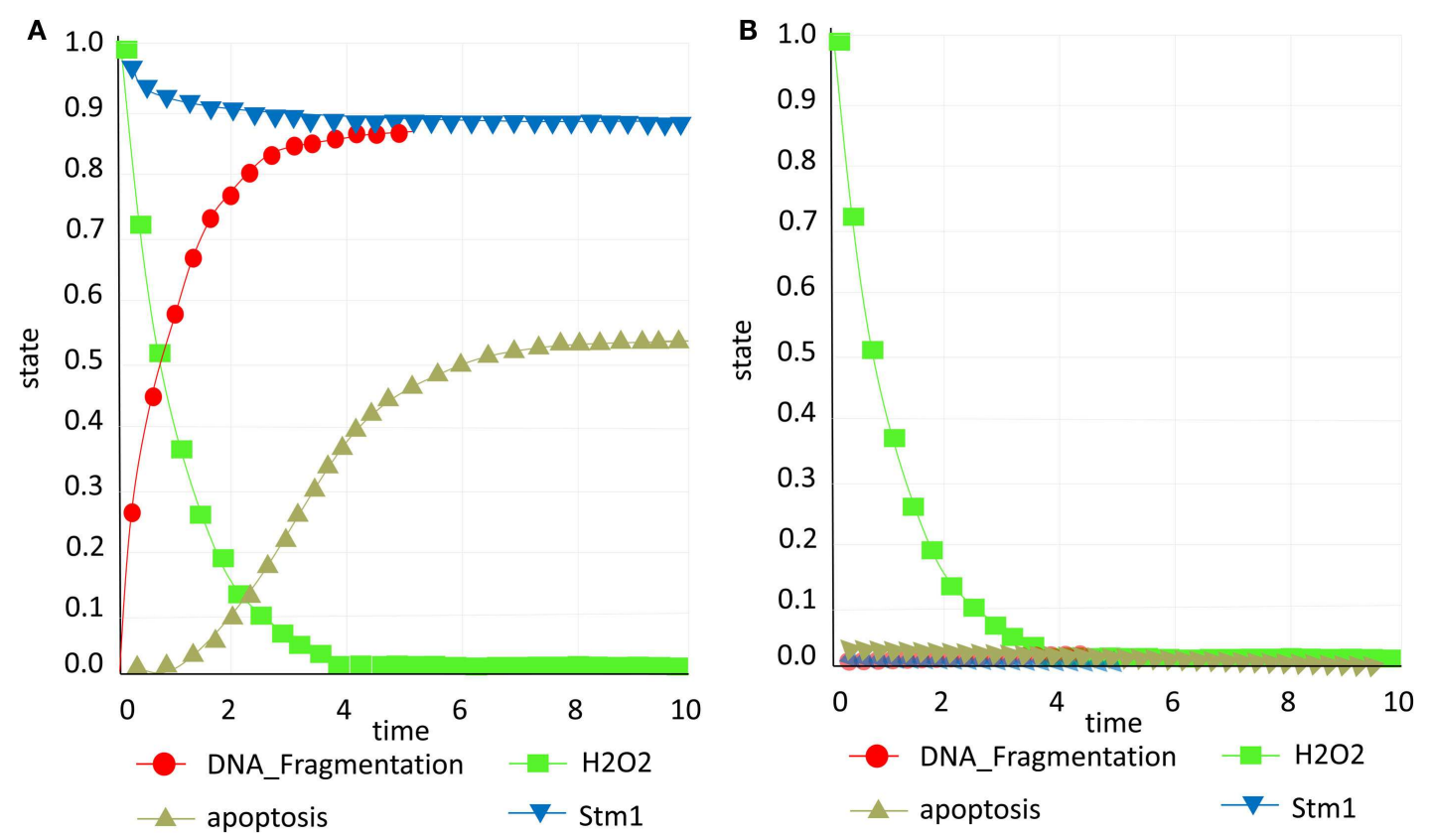

FIGURE 6 | Stm1 study. (A) Presence of Stm1p (blue) promotes apoptosis (yellow; B) Knock out of Stm1p (blue) prevents apoptosis (yellow) and DNA fragmentation (red) and consequently promotes survival.

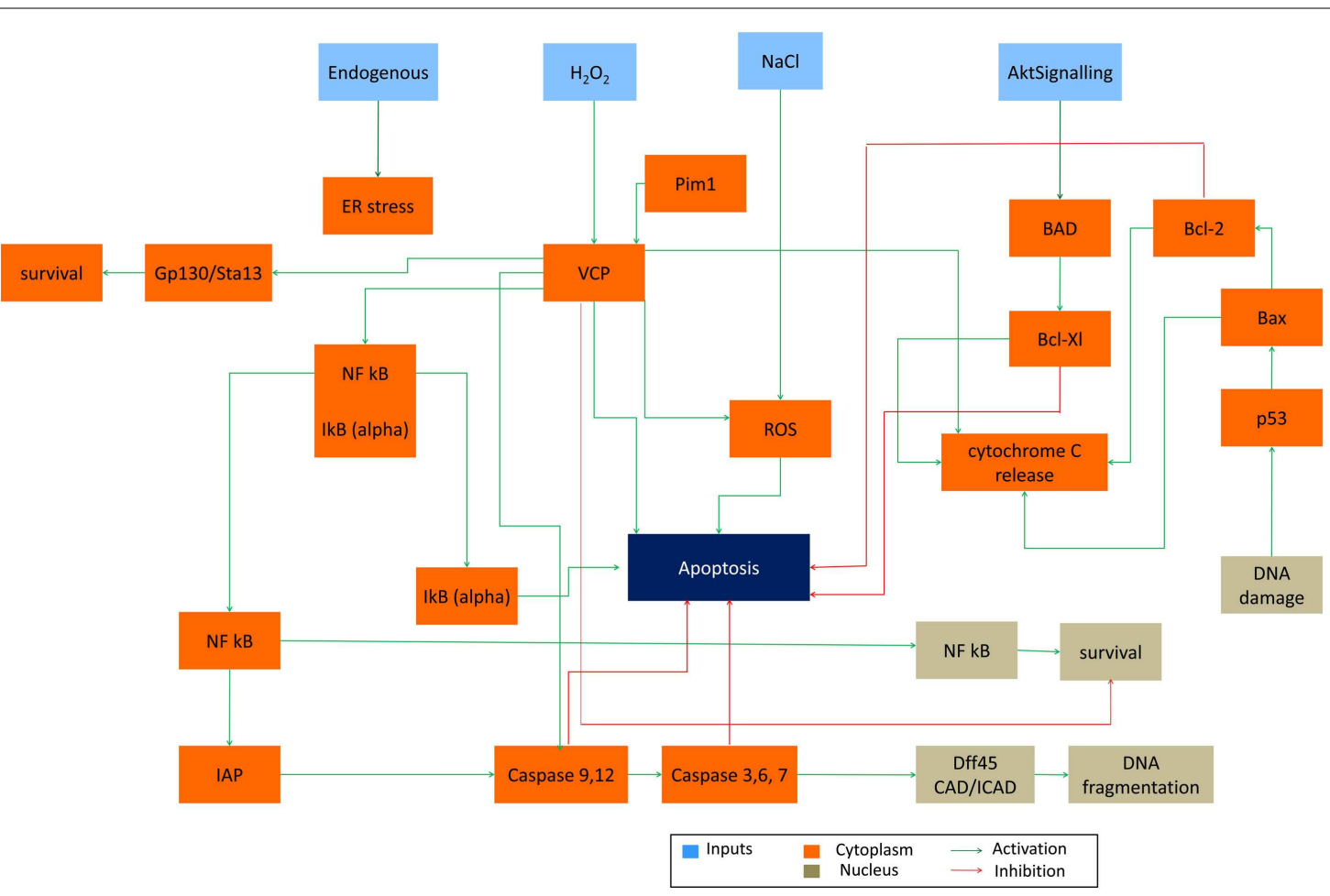

FIGURE 7 | In silico "humanized yeast apoptosis network". Human apoptotic pathways BCL-2 protein family and VCP dependent genes inserted into the yeast apoptosis network (due to simplicity we show only human pathways). 
Table 5 | Summary of simulations upon insertion of Bcl-2 pathway.

\begin{tabular}{lllll}
\hline Species & $\boldsymbol{t}=\mathbf{0}$ & $\boldsymbol{t}=\mathbf{4}$ & $\boldsymbol{t}=\mathbf{5}$ & $\boldsymbol{t}=\mathbf{6}$ \\
\hline UV & 0 & 0 & 0 & 0 \\
Akt signaling & 1 & 1 & 1 & 1 \\
BCL-XL & 0 & 1 & 1 & 1 \\
P53 & 0 & 0 & 0 & 0 \\
BAD & 0 & 0 & 0 & 0 \\
BAX & 0 & 0 & 0 & 0 \\
BCL-2 & 0 & 1 & 1 & 1 \\
Apoptosis & 0 & 0 & 0 & 0
\end{tabular}

Table includes simulation results of heterologous expression of BCL-2 in yeast apoptosis model. T stands for different time steps. Upon activation of Akt Signaling pathway at first time step BCL-2 gets activated in following step and as an inhibitor of apoptosis prevents apoptosis till end point.

good candidate for further investigation and exploration of model capabilities.

In our model, yeast Cdc48 was replaced with human VCP gene and its downstream pathway effector caspases (caspases 3, 6 and 7), initiator caspases (caspase9 and 12), IAP family, IkB-alpha inhibitory protein which when degraded by proteasome cause the release, and nuclear translocation of active NFkB (represented in the model as NFkB_Cyt and NFkB_Nuc) and gp130/Stat3 pathway (represented as a single node).

Upon activation of Akt Signaling pro-survival role of VCP is observed (node Survival $=1$ at $t=6$, Table 3 ). This is achieved when $\operatorname{IkB} \alpha$ is dissociated from $\mathrm{NFkB}$ (node $\mathrm{NFkB} / \mathrm{IkB} \alpha=1$ at $t=4$ ), which in turn gets activated (node $\mathrm{NFkB}=1, t=5$ ) and is translocated to the nucleus (nodes NFkB_Cyt $=1$ and NFkB_Nuc $=1, t=6$ ) inducing survival of the cell (node Survival $=1, t=6$ ). Simultaneously, activation of NFkB, promotes activity of IAP family of proteins which inhibits the activity of both effector and initiator caspases (nodes c-3-6-7 $=0$ and c-9-12 $=0$, $t=[0,4,5,6]$, thus disabling apoptosis-dependent caspase pathway. As an independent, but parallel process, activation of VCP via Akt Signaling activates gp130-Stat3 (node gp130-Stat3 $=1, t=4$ ) pathway and consequently leads to survival (Table 6).

This result suggests that yeast carrying mammalian VCP gene exhibits the same behavior as it is known from the human apoptotic model (Vandermoere et al., 2006).

\section{DISCUSSION}

In this work we have constructed a Boolean model for the biochemical network that controls apoptosis pathway in budding yeast Saccharomyces cerevisiae. Firstly, we presented the Boolean model describing only yeast pro and anti-apoptotic genes and validated the model by further analyzing the role of Stm1p, Birlp, and Hoglp. Even though construction of the yeast apoptosis network involved certain simplifications (nodes have only two states and rules are describing network dynamics) we were able to model more complex network than using dynamic modeling approach that requires knowledge of kinetic parameters for all molecular processes. This approach was able to suggest general design principals of yeast apoptosis. One of the advantages of constructing mathematical models in biology is that we are able to simulate
Table 6 | Summary of simulations upon insertion of VCP pathway.

\begin{tabular}{lllll}
\hline Species & $\boldsymbol{t}=\mathbf{0}$ & $\boldsymbol{t}=\mathbf{4}$ & $\boldsymbol{t}=\mathbf{5}$ & $\boldsymbol{t}=\mathbf{6}$ \\
\hline Akt signaling & 1 & 1 & 1 & 1 \\
GP130-STAT3 & 0 & 1 & 1 & 1 \\
NFkB-CYT & 0 & 1 & 1 & 1 \\
NFkB-NUC & 0 & 1 & 1 & 1 \\
NFkB/lkB $\alpha$ & 0 & 1 & 1 & 1 \\
VCP & 1 & 1 & 1 & 1 \\
C-3-6-7 & 0 & 0 & 0 & 0 \\
C-9-12 & 0 & 0 & 0 & 0 \\
IkB $\alpha$ & 0 & 1 & 1 & 1 \\
IAP & 0 & 1 & 1 & 1 \\
Survival & 0 & 0 & 0 & 1 \\
Apoptosis & 0 & 0 & 0 & 0
\end{tabular}

Table includes simulation results of humanized yeast apoptosis model by insertion of VCP. T stands for different time steps. VCP in presence of Akt Signaling is expected to prevent apoptosis and promote survival at last time point.

scenarios that are not feasible in real experiments. In the case of Bir $1 p$ we were able to in silico revive a cell already reaching apoptosis. These examples are important to understand general mechanisms of the constructed network. A study of proteasomal substrate Stm1p suggested that Stm1p seems to be an effector of $\mathrm{H}_{2} \mathrm{O}_{2}$ and the presence of Stmlp with an inducer leads to apoptosis in our model, and in Stm 1p knock out survival is promoted and DNA fragmentation is avoided, irrespective of the presence of the inducer. Despite the limitation of $\mathrm{BN}$ in terms of giving quantitative predictions of the system dynamics they allow investigation of the large networks and their systematic exploration resulting in better understanding of cellular processes. Example of Hoglp study showed that our model was capable of reproducing the pattern of Hog $1 \mathrm{p}$ activation, but was not able to quantitatively predict the maximal Hoglp activity.

In the second stage, we extended the initial model by inserting two pathways of human apoptosis (VCP and Bcl-2 family), hereby creating a "humanized" in silico yeast. Humanized yeast strains are used in experimental molecular biology where the human proteins (causing, or associated with diseases) are expressed and studied in vivo (in yeast in this case).

Conservation of many apoptotic mediators and mechanisms among the Eukarya provides the possibility of inserting genes from other organisms into yeast (in vivo) or in the yeast apoptotic network (in silico). We validated constructed humanized yeast model by insertion of Bcl-2 family which have been previously successfully expressed in yeast. The model was able to reproduce well-known pro and anti-apoptotic phenotypes confirming that yeast expressing Bax accumulated ROS and showed other apoptotic markers like DNA fragmentation. To test whether or not specific pathways playing role in neurodegeneration and cancer can be elucidated by our Boolean model and can we consequently take advantage of a simple system like yeast to explore hypothesis generated for higher organisms, we in silico expressed evolutionary conserved VCP and its downstream components in existing yeast apoptosis network. VCP is important player in cancer cell survival and can be used as a target for cancer therapy. It 
also serves as detector of aggregated proteins that are known to be cause of neurodegenerative diseases such as Parkinson's and Alzheimer's. Our model showed that cell survival is mediated by VCP via the degradation of $\operatorname{IkB} \alpha$ that leads to translocation of NFkB to nucleus. This result is in agreement with experimental findings that VCP is an essential target in the Akt signaling pathway suggesting that presented model in combination with experimental approach would represents a promising platform to study complex cellular processes involved in cancer and neurodegeneration.

Exploiting the advantages of BN models that enables extraction of system level properties of large networks and extensive state exploration and converting discrete model to continuous model our results suggested that in contrast to human apoptotic network yeast apoptosis is linear process whose regulation does not involve any complex feedback loops. Analysis of the second model showed that with certain adjustments Boolean model of yeast apoptosis can be adapted for studies of apoptosis in higher organisms.

Our results show that even without kinetic and qualitative data, it is possible to build models that can simulate relevant states of yeast physiology and regulations and can contribute to further understanding of biology. Since yeast is a preferred model organism for many studies of fundamental processes in a Eukaryal cell, we argue that in silico studies of yeast will be an important contributor to the understanding of complex cellular regulations, such as cell death pathways and that the applications will extend toward study of regulations or causes of human diseases such as cancer and neurodegeneration.

\section{MATERIALS AND METHODS \\ APOPTOSIS NETWORK SETUP}

Based on the extensive literature study and databases search ( Saccharomyces Genome Database-SGD; Cherry et al., 2011) the apoptosis network consisting of 73 nodes and 115 edges is constructed. These nodes are selected based on their interactions and their substrates involved in apoptosis. Cell Designer (Funahashi et al., 2003) was used to visualize genes and their connection. Cell Designer allowed us to represent network using comprehensive graphical notation. Moreover, Cell Designer is able to connect to online data bases such as KEGG (Kanehisa and Goto, 2000), BioModels (Li et al., 2010) and PubMed which expands its connectivity, visualization and model building. The most prominent advantage of Cell Designer is its ability to support SBML (Systems Biology Markup Language; Hucka et al., 2003) format. Creating SBML file avoids creating logic rule file which is more error prone when rules have to be defined by user in simple text and it is not in form of easily drawing boxes.

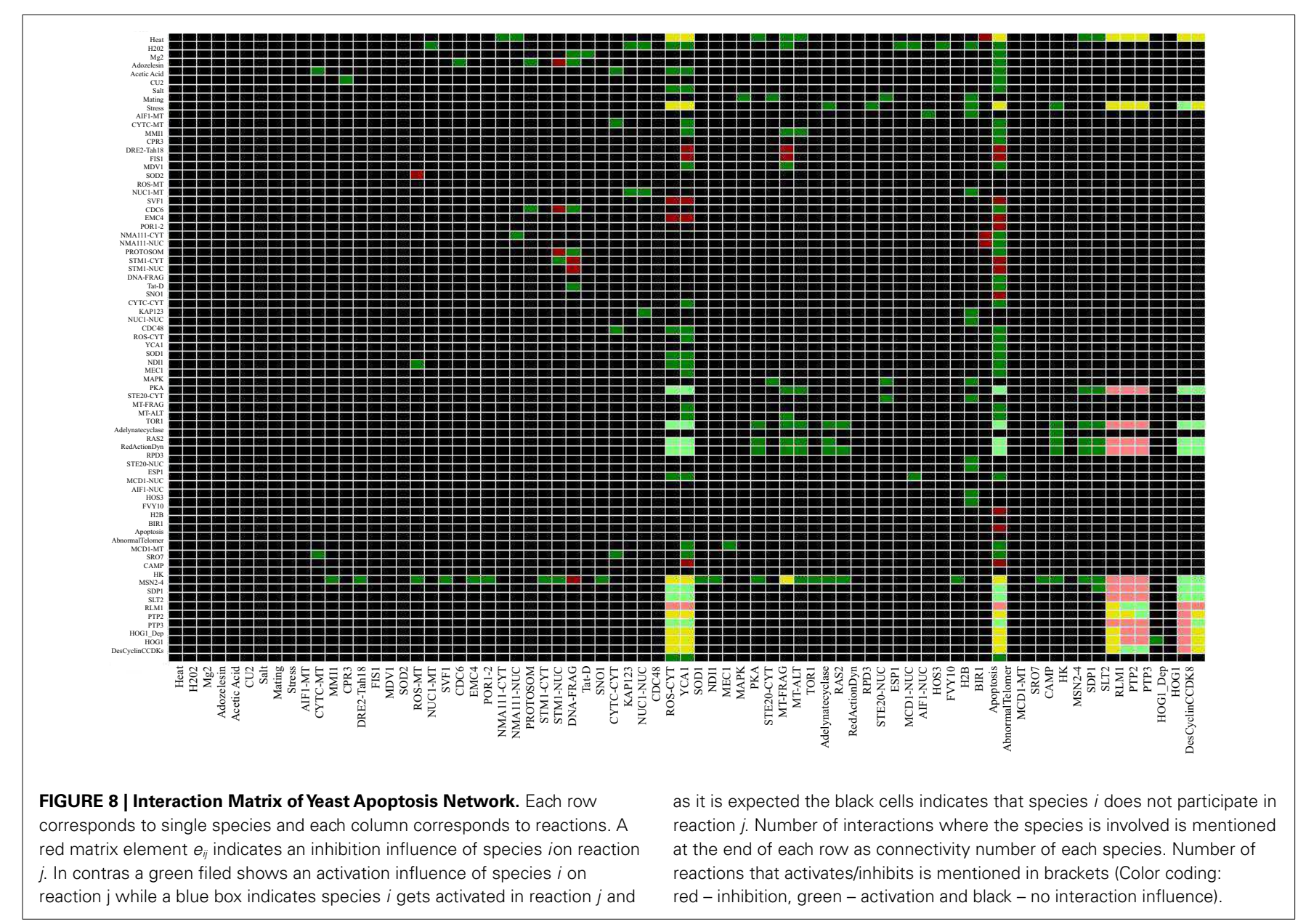




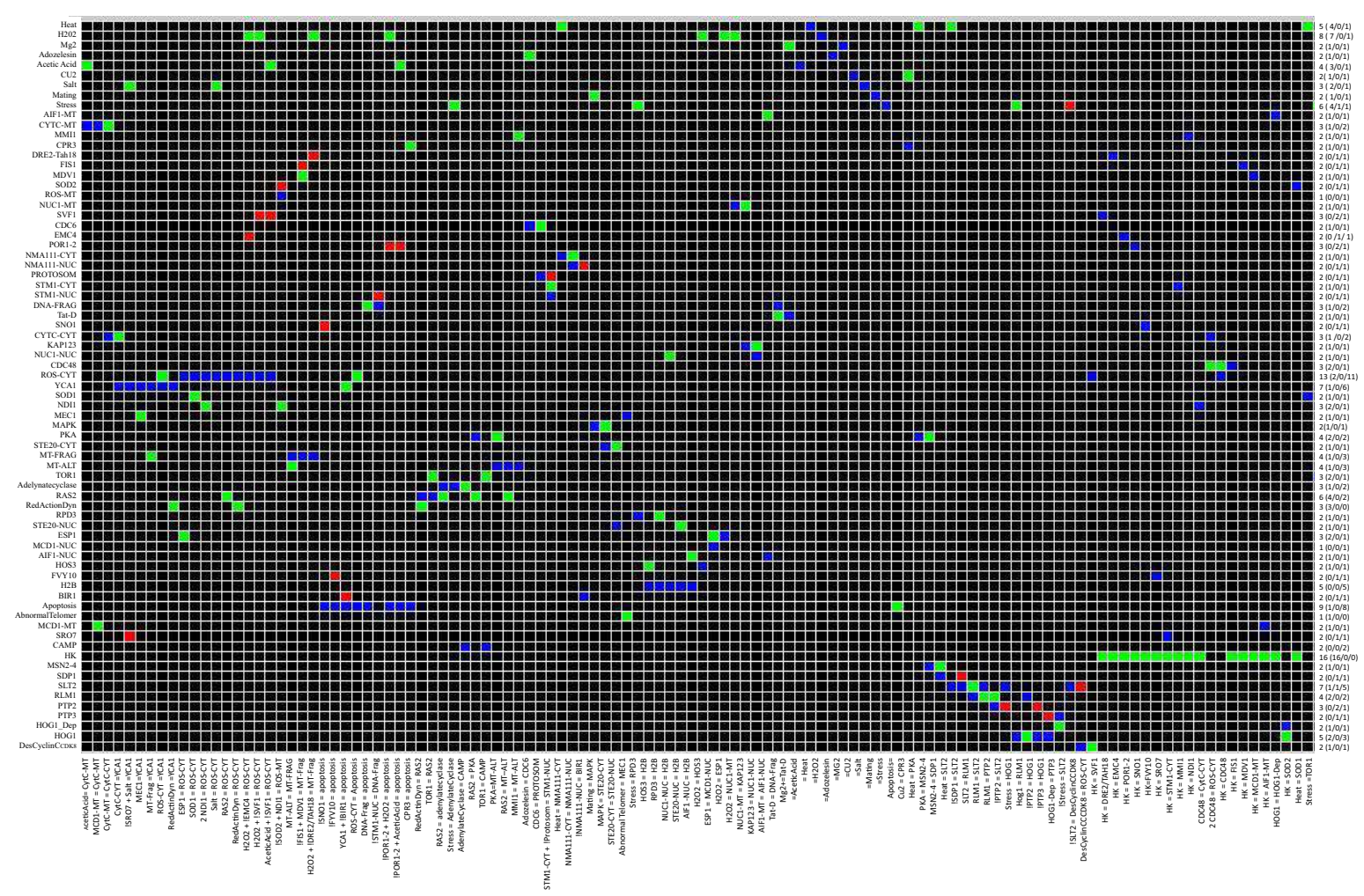

FIGURE 9 | Dependency Matrix for Yeast Apoptosis Network. In the dependency matrix each element $m_{i j}$ represent the relation between an affecting and an affected species. Former is specified at the bottom of each column and later is shown at the beginning of each row. At intersection of $i^{\text {th }}$ column and $j^{\text {th }}$ row there are three possibilities. A yellow box indicates species iis an ambivalent factor meaning both activating and inhibiting path exist from species $i$ to species $j$. Similarly a dark green and or light green cel shows a total and a non-total activator respectively. Another possibility is having dark or light red indicates species $i$ is a total inhibitor or non-total inhibitor of species $j$. whenever there is no path from spices $i$ to species $j$ the intersection cell is field by black. The hyper graph underlying the network is a directed graph and consequently the dependency matrix is non-symmetric. (Color coding: light and dark green - complete and incomplete activation, dark and light red - complete and incomplete inhibitor, yellow indicates an ambivalent factor and black indicates that there is no dependency between two species).

\section{COMPUTATION OF LOGICAL STEADY STATES}

Identification of Logical Steady States (LSSs) in a Boolean network is an important task as they comprise the states in which a gene-regulatory network resides most of the time. Strong biological implication can be carried out by LSSs of the network. LSSs can even be linked to phenotype (Kauffman, 1969). CNA (Klamt et al., 2006) is used to calculate all possible LLSs based on specified initial value for each gene and signal flow in network. LSSs are used to evaluate the network behavior under perturbation and changes in network structure.

\section{IDENTIFYING NETWORK WIDE DEPENDENCY}

Considering a pair of nodes $(a, b)$ dependency is defined as the influence of node $a$ on node $b$ and vice versa, node $a$ influences node $b$ as follow: a is $a$ total activator of $b$ if there is an activating path between $a$ and $b, a$ is a total inhibitor of $b$ is there is an inhibition path from $a$ to $b$ or a is an ambivalent effecter of $\mathrm{b}$ meaning that there is an intermediate node which is involved in a negative feedback loop. Using CNA the interaction matrix and dependency matrix were drawn from the network (Figures 8 and 9).

\section{CONVERSION OF DISCRETE TO CONTINUOUS MODEL USING SQUAD}

In order to convert the schematic network into Boolean model we used SQUAD (Mendoza and Xenarios, 2006), a user friendly graphical software which is suitable for modeling signaling network where kinetic reactions are not available. Simulation in SQUAD consists of three steps: (1) the network is first loaded from the SBML file. Components of the network are presented as nodes and value of each node represents the state of that node. SQUAD converts the network to discrete dynamic model. Using Boolean algorithms all steady sates in network are calculated. (2) network is converted to a continues dynamic model generating sets of ordinary differential equations (ODEs) and steady states achieved in pervious step. (3) SQUAD allows perturbation in order to understand the role of each node within the network.

Using Reduced Order Binary Decision Diagram (ROBDD), it is possible to calculate steady states (Di Cara et al., 2007). ROBDD is a memory efficient data structure which is widely used in electronic field and has been proven to work for large binary networks. Moreover, ROBDD computes steady states for large networks $(n>50)$ in matter of seconds. Another advantage of this algorithm is its ability to identify the cyclic steady states. These oscillating 
states are reachable when system identifies a cyclic pattern instead of one single state.

In the first step, schematic network is supplied to SQUAD resulting in a discrete network, generating a set of either cyclic or single steady states. These steady states are then used to convert discrete model to continues. Given all calculated steady states or cycles we examined all possible outcomes for each input. Depending on the desire form of output, discrete to continuous conversion can be carried out either as a complete or progressive mode. Our simulations are performed in the complete mode since cell undergoing apoptosis should die after certain time and it is expected to maintain a constant level of apoptosis or survival (in case when apoptosis is not activated) at the end of each run. As an opposite to complete mode, the progressive model allows the user to stop the simulation at any time even before reaching the steady states.

\section{CONVERSION OF DISCRETE TO CONTINUOUS MODEL USING ODEFY}

Reconstructing Boolean model of yeast apoptosis from qualitative knowledge never gives details about concentration of molecules

\section{REFERENCES}

Aerts, A. M., Zabrocki, P., François, I. E., Carmona-Gutierrez, D., Govaert, G., Mao, C., et al. (2008). Ydclp ceramidase triggers organelle fragmentation, apoptosis and accelerated ageing in yeast. Cell. Mol. Life Sci. 65, 1933-1942.

Ahn, S. H., Diaz, R. L., Grunstein, M., and Allis, C. D. (2006). Histone H2B deacetylation at lysine 11 is required for yeast apoptosis induced by phosphorylation of $\mathrm{H} 2 \mathrm{~B}$ at serine $10 . \mathrm{Mol}$. Cell 24, 211-220.

Albertyn, J., and Hohmann, S. (1994). Characterization of the osmotic-stress response in Saccharomyces cerevisiae: osmotic stress and glucose repression regulate glycerol-3-phosphate dehydrogenase independently. Curr. Genet. 25, 12-18.

Almeida, B., Silva, A., Mesquita, A. Sampaio-Marques, B., Rodrigues, F., and Ludovico, P. (2008). Druginduced apoptosis in yeast. Biochim. Biophys. Acta 7, 1436-1448.

Blanchard, F., Rusiniak, M. E., Sharma, K., Sun, X., Todorov, I., Castellano, M. M., et al. (2002). Targeted destruction of DNA replication protein Cdc6 by cell death pathways in mammals and yeast. Mol. Biol. Cell 13, 1536-1549.

Braun, R. J., and Zischka, H. (2008). Mechanisms of Cdc48/VCP-mediated cell death-from yeast apoptosis to human disease. Biochim. Biophys. Acta 1783, 1418-1435.

Brewster, J. L., de Valoir, T., Dwyer, N. D, Winter, E., and Gustin, M. C. (1993). An osmosensing signal transduction pathway in yeast. Science 259, 1760-1763.
Buttner, S., Eisenberg, T., CarmonaGutierrez, D., Ruli, D., Knauer, H., Ruckenstuhl, C., et al. (2007). Endonuclease $\mathrm{G}$ regulates budding yeast life and death. Mol. Cell 25, 233-246.

Carmona-Gutierrez, D., Eisenberg, T., Büttner, S., Meisinger, C., Kroemer, G., and Madeo, F. (2010a). Apoptosis in yeast: triggers, pathways, subroutines. Cell. Death Differ. 17, 763-773.

Carmona-Gutierrez, D., Ruckenstuhl, C., Bauer, M. A., Eisenberg, T., Buttner, S., and Madeo, F. (2010b). Cell death in yeast: growing applications of a dying buddy. Cell Death Differ. 17, 733-734.

Cherry, J. M., Hong, E. L., Amundsen, C., Balakrishnan, R., Binkley, G., Chan, E. T., et al. (2011). Saccharomyces genome database: the genomics resource of budding yeast. Nucleic Acids Res. 40, D700-D705. [Database issue]

Datta, S. R., Dudek, H., Tao, X., Masters, S., Fu, H., Gotoh, Y., et al. (1997). Akt phosphorylation of BAD couples survival signals to the cellintrinsic death machinery. Cell 91, 231-241.

Davidich, M., and Bornholdt, S. (2008). Boolean network model predicts cell cycle sequence of fission yeast. PLoS ONE 3:e1672. doi:10.1371/journal.pone.0001672

de Nadal, E., Zapater, M., Alepuz, P. M., Sumoy, L., Mas, G., and Posas, P. (2004). The MAPK Hog1 recruits Rpd3 histone deacetylase to activate osmoresponsive genes. Nature 427, 370-374. G., Xenarios, I., and Mendoza, L. (2007). Dynamic simulation
Di Cara, A., Garg, A., De Micheli,

in different time points. For this purpose the discrete Boolean model is transformed to continuous model using Odefy. Odefy uses the multivariate polynomial interpolation in order to transform the logical rules into sets of ODEs. Yeast apoptosis Boolean model is converted to continuous model using Hill Cube and normalized Hill Cube where the Hill function is normalized to the unit interval. Behavior of biochemical reactions can be seen as a sigmoid Hill function represented as $f(\overline{\bar{x}})=\overline{\bar{x}}^{n} /\left(\overline{\bar{x}}^{n}+k^{n}\right)$. Where, $n$ is a Hill coefficient and determines the slope of the curve and is a measurement of cooperativity of the interactions, and parameter $k$ corresponds to values 1 and 0 in the Boolean model in the following manner: threshold value above given $k$ in Boolean model is considered as 1 (on state) and below as 0 (off state).

\section{ACKNOWLEDGMENTS}

We would like to thank Dr. Andrea Molt for initial discussions and the Knut and Alice Wallenberg Foundation, the Chalmers Foundation, and the FP7 EU Project SYSINBIO for funding.

of regulatory networks using SQUAD. BMC Bioinformatics 8:462. doi:10.1186/1471-2105-8-462

Drexler, H. C. A. (1997). Activation of the cell death program by inhibition of proteasome function. Proc. Natl. Acad. Sci. U.S.A. 94, 855-860.

Eisenberg, T., Buttner, S., Kroemer, G., and Madeo, F. (2007). The mitochondrial pathway in yeast apoptosis. Apoptosis 12, 1011-1023.

Fahrenkrog, B., Sauder, U., and Aebi, U. (2004). The S. cerevisiae HtrAlike protein Nmall1p is a nuclear serine protease that mediates yeast apoptosis. J. Cell Sci. 117, 115-126.

Falasca, M. (2010). PI3K/Akt signalling pathway specific inhibitors: a novel strategy to sensitize cancer cells to anti-cancer drugs. Curr. Pharm. Des. 16, 1410-1416.

Fauré, A., Naldi, A., Chaouiya, C., and Thieffry, D. (2006). Dynamic analysis of a generic Boolean model for the control of the mammalian cell cycle. Bioinformatics 22, 124-131.

Ferrigno, P., Posas, F., Koepp, D., Saito, H., and Silver, P. A. (1998). Regulated nucleo/cytoplasmic exchange of HOG1 MAPK requires the importin beta homologs NMD5 and XPO1. EMBO J. 17, 5606-5614.

Frohlich, K. U., Fussi, H., and Ruckenstuhl, C. (2007). Yeast apoptosisfrom genes to pathways. Semin. Cancer Biol. 17, 112-121.

Funahashi, A., Tanimura, N., Matsuoka, Y., Yosinaga, N., and Kitano, H. (2003). CellDesigner: a process diagram editor for gene-regulatory and biochemical networks. BIOSILICO 1, 159-162.

Garbarino, J., Padamsee, M., Wilcox, L., Oelkers, P. M., D’ Ambrosio,
D., Ruggles, K., et al. (2009). Sterol and diacylglycerol acyltransferase deficiency triggers fatty acidmediated cell death. J. Biol. Chem. 284, 30994-31005.

Greenhalf, W., Stephan, C., and Chaudhuri, B. (1996). Role of mitochondria and $\mathrm{C}$-terminal membrane anchor of Bcl-2 in Bax induced growth arrest and mortality in Saccharomyces cerevisiae. FEBS Lett. 380, 169-175.

Gupta, S., Bisht, S. S., Kukreti, R., Jain, S., and Brahmachari, S. K. (2007). Boolean network analysis of neurotransmitter signaling pathway. $J$. Theor. Biol. 244, 463-469.

Hahn, J. S., and Thiele, D. J. (2002). Regulation of the Saccharomyces cerevisiae Slt2 kinase pathway by the stress-inducible Sdp1 dual specificity phosphatase. J. Biol. Chem. 277, 21278-21284.

Handorf, T., and Klipp, E. (2012). Modeling mechanistic biological networks: an advanced Boolean approach. Bioinformatics 28 , 557-563.

Hauptmann, P., Riel, C., KunzSchughart, L. A., Fröhlich, K. U., Madeo, F., and Lehle, L. (2006). Defects in $\mathrm{N}$-glycosylation induce apoptosis in yeast. Mol. Microbiol. 59, 765-778.

Hers, I., Vincent, E. E., and Tavaré, J. M. (2011). Akt signalling in health and disease. Cell. Signal. 23, 1515-1527.

Hirabayashi, M., Inoue, K., Tanaka, K., Nakadate, K., Ohsawa, Y., Kamei, Y., et al. (2001). VCP/p97 in abnormal protein aggregates, cytoplasmic vacuoles, and cell death, phenotypes relevant to neurodegeneration. Cell Death Differ. 8, 977-984. 
Hohmann, S. (2002). Osmotic stress signaling and osmoadaptation in yeast. Microbiol. Mol. Biol. Rev. 66, 300-372.

Hohmann, S. (2009). Control of high osmolarity signalling in the yeast Saccharomyces cerevisiae. FEBS Lett. 583, 4025-4029.

Hucka, M., Finney, A., Sauro, H. M., Bolouri, H., Doyle, J. C., and Kitano, H. (2003). The systems biology markup language (SBML): a medium for representation and exchange of biochemical network models. Bioinformatics 19, 524-531.

Imamuraab, S., Ojimaa, N., and Yamashitaa, M. (2003). Coldinducible expression of the cell division cycle gene CDC48 and its promotion of cell proliferation during cold acclimation in zebrafish cells. FEBS Lett. 549, 14-20.

Ishigaki, S., Hishikawa, N., Niwa, J., Iemura, S., Natsume, T., Hori, S., et al. (2004). Physical and functional interaction between dorfin and valosin-containing protein that are colocalized in ubiquitylated inclusions in neurodegenerative disorders. J. Biol. Chem. 279, 51376-51385.

Janes, K. A., Albeck, J. G., Sorger, S. G. P. K., Lauffenburger, D. A., and Yaffe, M. B. (2005). A systems model of signaling identifies a molecular basis set for cytokine-induced apoptosis. Science 310, 1646-1653.

Kanehisa, M., and Goto, S. (2000). KEGG: kyoto encyclopedia of genes and genomes. Nucleic Acids Res. 28, 27-30.

Kataoka, T., Powers, S., McGill, C., Fasano, O., Strathern, J., Broach, J., et al. (1984). Genetic analysis of yeast RAS1 and RAS2 genes. Cell 37, 437-445.

Kauffman, S. A. (1969). Metabolic stability and epigenesis in randomly constructed genetic nets. J. Theor. Biol. 22, 437-467.

Kharbanda, S., Pandey, P., Schofield, L., Israels, S., Roncinske, R., Yoshida, K., et al. (1997). Role for Bcl-xL as an inhibitor of cytosolic cytochrome $\mathrm{C}$ accumulation in DNA damageinduced apoptosis. Proc. Natl. Acad. Sci. U.S.A. 13, 6939-6942.

Khoury, C. M., Yang, Z., Li, X. Y., Vignali, M., Fields, S., and Greenwood, M. T. (2008). A TSC22-like motif defines a novel antiapoptotic protein family. FEMS Yeast Res. 8, 540-563.

Klamt, S., Saez-Rodriguez, J., Lindquist, J. A., Simeoni, L., and Gilles, E. D. (2006). A methodology for the structural and functional analysis of signaling and regulatory networks. BMC Bioinformatics 7:56. doi:10.1186/1471-2105-7-56

Krasley, E., Cooper, K. F., Mallory, M. J., Dunbrack, R., and Strich, R. (2006). Regulation of the oxidative stress response through Slt2p-dependent destruction of cyclin $\mathrm{C}$ in Saccharomyces cerevisiae. Genetics 172, 1477-1486.

Krumsiek, J., Pölsterl, S., Wittmann, D. M., and Theis, F. J. (2010). Odefy - from discrete to continuous models. BMC Bioinformatics 11:233. doi:10.1186/1471-2105-11-233

Li, C., Donizelli, M., Rodriguez, N., Dharuri, H., Endler, L., Chelliah, V., et al. (2010). BioModels Database: an enhanced, curated and annotated resource for published quantitative kinetic models. BMC Syst. Biol. 4:92. doi:10.1186/1752-0509-4-92

Li, W., Sun, L., Liang, Q., Wang, J., Mo, W., and Zhou, B. (2006). Yeast AMID homologue Ndilp displays respiration-restricted apoptotic activity and is involved in chronological aging. Mol. Biol. Cell 17, 1802-1811.

Liang, Q., and Zhou, B. (2007). Copper and manganese induce yeast apoptosis via different pathways. Mol. Biol. Cell 18, 4741-4749.

Ligr, M., Velten, I., Fröhlich, E., Madeo, F., Ledig, M., Fröhlich, K. U., et al. (2001). The proteasomal substrate Stml participates in apoptosis-like cell death in yeast. Mol. Biol. Cell 12, 2422-2432.

Low, C. P., Shui, G., Liew, L. P., Buttner, S., Madeo, F., Dawes, I. W., et al. (2008). Caspase-dependent and -independent lipotoxic cell-death pathways in fission yeast. J. Cell. Sci. 15, 2671-2684.

Ludovico, P., Rodrigues, F., Almeida, A., Silva, M. T., Barrientos, A., and Côrte-Real, M. (2002). Cytochrome c release and mitochondria involvement in programmed cell death induced by acetic acid in Saccharomyces cerevisiae. Mol. Biol. Cell 13, 2598-2606.

Ludovico, P., Sousa, M. J., Silva, M. T., Leao, C., and Côrte-Real, M. (2001). Saccharomyces cerevisiae commits to a programmed cell death process in response to acetic acid. Microbiology 147, 2409-2415.

Madeo, F., Carmona-Gutierrez, D., Ring, J., Buttner, S., Eisenberg, T., and Kroemer, G. (2009). Caspase-dependent and caspaseindependent cell death pathways in yeast. Biochem. Biophys. Res. Commun. 382, 227-231.

Madeo, F., Fröhlich, E., and Fröhlich, K. U. (1997). A yeast mutant showing diagnostic markers of early and late apoptosis. J. Cell Biol. 139, 729-734.

Madeo, F., Fröhlich, E., Ligr, M., Grey, M., Sigrist, S. J., Wolf, D. H., et al. (1999). Oxygen stress: a regulator of apoptosis in yeast. J. Cell Biol. 145, 757-767.

Madeo, F., Herker, E., Maldener, C., Wissing, S., Lächelt, S., Herlan, M., et al. (2002). A caspase-related protease regulates apoptosis in yeast. Mol. Cell 9, 911-917.

Mai, Z., and Liu, H. (2009). Boolean network-based analysis of the apoptosis network: irreversible apoptosis and stable surviving. J. Theor. Biol. 259, 760-769.

Manon, S., Chaudhuri, B., and Guérin, M. (1997). Release of cytochrome $c$ and decrease of cytochrome $c$ oxidase in Bax-expressing yeast cells, and prevention of these effects by coexpression of Bcl-xL. FEBS Lett. 415, 29-32.

Mazzoni, C., Herker, E., Palermo, V., Jungwirth, H., Eisenberg, T., Madeo, F., et al. (2005). Yeast caspase 1 links messenger RNA stability to apoptosis in yeast. EMBO Rep. 6 , 1076-1081.

Mendoza, L., and Xenarios, I. (2006). A method for the generation of standardized qualitative dynamical systems of regulatory networks. Theor Biol. Med. Model. 3, 13.

Mizuno, Y., Hori, S., Kakizuka, A. and Okamoto, K. (2003). Vacuolecreating protein in neurodegenerative diseases in humans. Neurosci. Lett. 343, 77-80.

Nelson, L. D., Musso, M., and Van Dyke, M. W. (2000). The yeast STM1 gene encodes a purine motif triple helical DNA-binding protein. J. Biol. Chem. 275, 5573-5581.

Owsianowski, E., Walter, D., and Fahrenkrog, B. (2008). Negative regulation of apoptosis in yeast. Biochim. Biophys. Acta 1783, 1303-1310.

Pereira, C., Camougrand, N., Manon, S., Sousa, M. J., and Côrte-Real, M. (2007). ADP/ATP carrier is required for mitochondrial outer membrane permeabilization and cytochrome c release in yeast apoptosis. Mol. Microbiol. 66, 571-582.

Qiu, J., Yoon, J. H., and Shen, B. (2005). Search for apoptotic nucleases in yeast: role of Tat-D nuclease in apoptotic DNA degradation. J. Biol. Chem. 280, 15370-15379.

Ring, G., Khoury, C. M., Solar, A. J., Yang, Z., Mandato, C. A., and Greenwood, M. T. (2008). Transmembrane protein 85 from both human (TMEM85) and yeast (YGL231c) inhibit hydrogen peroxide mediated cell death in yeast. FEBS Lett. 582, 2637-2642.

Ruchaud, S., Carmena, M., and Earnshaw, W. C. (2007). Chromosomal passengers: conducting cell division. Nat. Rev. Mol. Cell Biol. 8, 798-812.

Sato, T., Hanada, M., Bodrug, S., Irie, S., Iwama, N., Boise, L. H., et al. (1994). Interactions among members of the Bcl-2 protein family analyzed with a yeast two hybrid system. Proc. Natl. Acad. Sci. U.S.A. 91, 9238-9242.

Schlatter, R., Schmich, K., Vizcarra, I. A. Scheurich, P., Sauter, T., Borner, C. et al. (2009). On/off and beyond a boolean model of apoptosis. PLoS Comput. Biol. 5:e1000595. doi:10.1371/journal.pcbi.1000595

Schmelzle, T., Beck, T., Martin, D. E., and Hall, M. N. (2004). Activation of the RAS/cyclic AMP pathway suppresses a TOR deficiency in yeast. Mol. Cell Biol. 24, 338-351.

Song, C., Wang, Q., and Li, C. C. (2003). ATPase activity of p97valosin-containing protein (VCP). D2 mediate the major enzyme activity and D1 contributes in heatinduced activity. J. Biol. Chem. 278, 3648-3655.

Stelling, J. (2004). Mathematical models in microbial systems biology. Curr. Opin. Microbiol. 7, 513-518.

Thomas, R., and D'Ari, R. (1990). Biological Feedback. Boca Raton: CRC Press.

Uren, A. G., Coulson, E. J., and Vaux, D. L. (1998). Conservation of baculovirus inhibitor of apoptosis repeat proteins (BIRPs) in viruses, nematodes, vertebrates and yeasts. Trends Biochem. Sci. 23, 159-162.

Van Wuytswinkel, O., Reiser, V., Siderius, M., Kelders, M. C., Ammerer, G., Ruis, H., et al. (2000). Response of Saccharomyces cerevisiae to severe osmotic stress: evidence for a novel activation mechanism of the HOG MAP kinase pathway. Mol. Microbiol. 37, 382-397.

Vander Heiden, M. G., Choy, J. S., VanderWeele, D. J., Brace, J. L., Harris, M. H., Bauer, D. E., et al. (2002). Bcl$\mathrm{x}(\mathrm{L})$ complements Saccharomyces cerevisiae genes that facilitate the switch from glycolytic to oxidative metabolism. J. Biol. Chem. 277, 44870-44876

Vandermoere, F., El Yazidi-Belkoura, I., Slomianny, C., Demont, Y., Bidaux, G., Adriaenssens, E., et al. (2006). The valosin-containing protein (VCP) is a target of Akt signaling required for cell survival. J. Biol. Chem. 20, 14307-14313. 
Vernis, L., Facca, C., Delagoutte, E., Soler, N., Chanet, R., Guiard, B., et al. (2009). A newly identified essential complex, Dre2-Tah18, controls mitochondria integrity and cell death after oxidative stress in yeast. PLoS ONE 4:e4376. doi:10.1371/journal.pone.0004376

Wadskog, I., Maldener, C., Proksch, A., Madeo, F., and Adler, L. (2004). Yeast lacking the SRO7/SOP1-encoded tumor suppressor homologue show increased susceptibility to apoptosislike cell death on exposure to $\mathrm{NaCl}$ stress. Mol. Biol. Cell 15, 1436-1444.

Walter, D., Wissing, S., Madeo, F., and Fahrenkrog, B. (2006). The inhibitor-of-apoptosis protein Birlp protects against apoptosis in $\mathrm{S}$. cervisiae and is a substrate for the yeast homologue of Omi/HtrA2. J. Cell. Sci. 119, 1843-1851.

Wang, Q., Song, C., and Li, C. H. (2004). Molecular perspective on p97-VCP: progress in understanding its structure and diverse biological function. J. Struct. Biol. 164, 44-57.

Warmka, J., Hanneman, J., Lee, J., Amin, D., and Ota, I. (2001). Ptcl, a type 2C Ser/Thr phosphatase, inactivates the HOG pathway by dephosphorylating the mitogen-activated protein kinase Hog1. Mol. Cell. Biol. 21, 51-60.

Weinberger, M., Ramachandran, L., Feng, L., Sharma, K., Sun, X., Marchetti, M., et al. (2005). Apoptosis in budding yeast caused by defects in initiation of DNA replication. J. Cell Sci. 118, 3543-3553.

Westfall, P. J., Ballon, D. R., and Thorner, J. (2004). When the stress of your environment makes you go HOG wild. Science 306, 1511-1512.

Winderickx, J., Delay, C., De Vos, A., Klinger, H., Pellens, K., Vanhelmont, T., et al. (2008). Protein folding diseases and neurodegeneration: lessons learned from yeast. Biochim. Biophys. Acta 1783, 1381-1395.

Winkler, A., Arkind, C., Mattison, C. P., Burkholder, A., Knoche, K., and Ota, I. (2002). Heat stress activates the yeast high-osmolarity glycerol mitogen-activated protein kinase pathway, and protein tyrosine phosphatases are essential under heat stress. Eukaryot. Cell 1, 163-173.

Wissing, S., Ludovico, P., Herker, E., Buttner, S., Engelhardt, S. M., Decker, T., et al. (2004). An AIF orthologue regulates apoptosis in yeast. J. Cell Biol. 166, 969-974.

Wittmann, D. M., Krumsiek, J., SaezRodriguez, J., Lauffenburger, D. A.,
Klamt, S., and Theis, F. J. (2009). Transforming Boolean models to continuous models: methodology and application to T-cell receptor signaling. BMC Syst. Biol. 3:98. doi:10.1186/1752-0509-3-98

Wolkenhauer, O. (2002). Mathematical modelling in the post-genome era: under-standing genome expression and regulation - a system theoretic approach. BioSystems 65 $1-18$.

Wood, D. R., Poullet, P., Wilson, B. A., Khalil, M., Tanaka, K., Cannon, J. F., et al. (1994). Biochemical characterization of yeast RAS2 mutants reveals a new region of ras protein involved in the interaction with GTPase-activating proteins. J. Biol. Chem. 269, 5322-5327.

Yang, H., Ren, Q., and Zhang, Z. (2008). Cleavage of Mcdl by caspase-like protease Esp1 promotes apoptosis in budding yeast. Mol. Biol. Cell 19, 2127-2134.

Young, C., Mapes, J., Hanneman, J., AlZarban, S., and Ota, I. (2002). Role of Ptc2 type 2C Ser/Thr phosphatase in yeast high-osmolarity glycerol pathway inactivation. Eukaryot. Cell 1, 1032-1040

Zhang, N. N., Dudgeon, D. D., Paliwal, S., Levchenko, A., Grote, E. and Cunningham, K. W. (2006).
Multiple signaling pathways regulate yeast cell death during the response to mating pheromones. Mol. Biol. Cell 17, 3409-3422.

Conflict of Interest Statement: The authors declare that the research was conducted in the absence of any commercial or financial relationships that could be construed as a potential conflict of interest.

Received: 29 June 2012; accepted: 07 November 2012; published online: 10 December 2012.

Citation: Kazemzadeh L, Cvijovic $M$ and Petranovic D (2012) Boolean model of yeast apoptosis as a tool to study yeast and human apoptotic regulations. Front. Physio. 3:446. doi: 10.3389/fphys.2012.00446

This article was submitted to Frontiers in Systems Biology, a specialty of Frontiers in Physiology.

Copyright (c) 2012 Kazemzadeh, Cvijovic and Petranovic. This is an openaccess article distributed under the terms of the Creative Commons Attribution License, which permits use, distribution and reproduction in other forums, provided the original authors and source are credited and subject to any copyright notices concerning any third-party graphics etc.
Research Topic:

From structural to molecular systems biology: experimental and computational approaches to unravel mechanisms of kinase activity regulation in cancer and neurodegeneration
Topic Editor:

Matteo Barberis,

Humboldt University Berlin, Germany;

Max Planck Institute for Molecular Genetics, Berlin, Germany 